OPEN ACCESS

Edited by: Dominik Wolf, Innsbruck Medical University, Austria

Reviewed by:

Yongfeng $\mathrm{He}$,

Weill Cornell Medicine, United States

Chao Wang,

Peking University People's

Hospital, China

*Correspondence:

Tian Li

tian@fmmu.edu.cn;

fmmult@foxmail.com

Lingyun Xia

xialingyun200810@163.com

${ }^{t}$ These authors have contributed equally to this work

Specialty section: This article was submitted to Gastrointestinal Cancers, a section of the journal

Frontiers in Oncology

Received: 04 March 2021 Accepted: 08 November 2021 Published: 29 November 2021

Citation:

Sun M, Ding H, Zhu Z, Wang S, Gu X, $X i a L$ and $L i T$ (2021) Identifying Optimal Surgical Intervention-Based Chemotherapy for Gastric Cancer Patients With Liver Metastases.

Front. Oncol. 11:675870. doi: 10.3389/fonc.2021.675870

\section{Identifying Optimal Surgical Intervention-Based Chemotherapy for Gastric Cancer Patients With Liver Metastases}

\author{
Min Sun ${ }^{1,2,3+}$, Hangliang Ding ${ }^{4 \dagger}$, Zhiqiang Zhu ${ }^{5 \dagger}$, Shengsheng Wang ${ }^{1}$, Xinsheng $\mathrm{Gu}^{6}$, \\ Lingyun $\mathrm{Xia}^{7^{*}}$ and Tian $\mathrm{Li}^{1,8^{*}}$
}

${ }^{1}$ Department of General Surgery, Taihe Hospital, Hubei University of Medicine, Shiyan, China, ${ }^{2}$ Department of Anesthesiology, Institute of Anesthesiology, Taihe Hospital, Hubei University of Medicine, Shiyan, China, ${ }^{3}$ Hubei Key Laboratory of Embryonic Stem Cell Research, Taihe Hospital, Hubei University of Medicine, Shiyan, China, ${ }^{4}$ Department of General Surgery, Xinchang Hospital Affiliated to Wenzhou Medical University, Wenzhou, China, ${ }^{5}$ Department of Pediatrics, Zhongnan Hospital of Wuhan University, Wuhan, China, ${ }^{6}$ College of Basic Medical Sciences, Hubei University of Medicine, Shiyan, China, ${ }^{7}$ Department of Stomatology, Taihe Hospital, Hubei University of Medicine, Shiyan, China, ${ }^{8}$ School of Basic Medicine, Fourth Military Medical University, Xi'an, China

Background: This study aimed at evaluating the effects of surgical treatments-based chemotherapy in the treatment of gastric cancer with liver metastases (GCLM). It has not been established whether Liver-directed treatment (LDT) options such as hepatectomy and gastrectomy plus chemotherapy (HGCT), radiofrequency ablation and gastrectomy plus chemotherapy (RFAG), transarterial chemoembolization and gastrectomy plus chemotherapy (TACEG), gastrectomy plus chemotherapy (GCT) enhance the survival of GCLM patients.

Methods: We performed systematic literature searches in PubMed, EMBASE, and Cochrane library from inception to September 2021. We created a network plot to comprehensively analyze the direct and indirect evidence, based on a frequentist method. A contribution plot was used to determine inconsistencies, a forest plot was used to evaluate therapeutic effects, the publication bias was controlled by funnel plot, while the value of surface under the cumulative ranking curves (SUCRA) was calculated to estimate rank probability.

Results: A total of 23 retrospective studies were identified, involving 5472 GCLM patients. For OS and 1-, 2-, 3-year survival rate of all trials, meta-analysis of the direct comparisons showed significant better for HGCT treatments compared with GCT or PCT. In the comparison of the 5 treatments for 1-, 2-, 3-year survival rate, HGCT and RFAG were found to be more effective than GCT and PCT, respectively. By OS and 2-, 3-year survival rate analysis, RFAG was identified as the best option, followed by HGCT, TACEG, GCT and PCT. By 1-year survival rate analysis, HGCT and RFAG were identified as the most effective options. 


\begin{abstract}
Conclusion: HGCT and RFAG has remarkable survival benefits for GCLM patients when compared to TACEG, GCT and PCT. HGCT was found to exhibit superior therapeutic effects for GCLM patients for 1-year survival rate while RFAG was found to be a prospective therapeutic alternative for OS and 2-, 3-year survival rate.
\end{abstract}

Systematic Review Registration: identifier [10.37766/inplasy2020.12.0009].

Keywords: gastric cancer, liver metastasis, hepatectomy, interventional therapy, network analysis

\section{INTRODUCTION}

Globally, gastric cancer is the fourth most common malignant tumor and the second highest cause of cancer-related mortalities (1-3). Therapeutic options for advanced gastric cancers have been enormously improved. In the last two decades, the 5-year survival rate is up to $40 \%$. However, gastric cancer with liver metastases is considered a late-stage disease. Systemic chemotherapy was recommended as standard cure, with a 5-year survival rate of less than $10 \%(4,5)$. The current standard management of GCLM is systemic chemotherapy with supportive care. Liver metastasis is a common phenomenon for many types of cancer (6-8). Liverdirected treatment (LDT) options such as hepatectomy and gastrectomy plus chemotherapy (HGCT), radiofrequency ablation and gastrectomy plus chemotherapy (RFAG), transarterial chemoembolization and gastrectomy plus chemotherapy (TACEG), gastrectomy plus chemotherapy (GCT) for GCLM is controversial $(5,9,10)$. Compared to systemic chemotherapy, surgical treatment such as HGCT and RFAG of hepatic metastases presents favorable prognosis (11-13). According to the guidelines of The Committee of the Japan Gastric Cancer Association (JGCA) and National Comprehensive Cancer Network (NCCN), palliative management is recommended for stage IV gastric cancer, e.g. GCLM. In contrast, colorectal liver metastases are considered as suitable targets for radical surgery because they often present as liveronly metastatic disease, and R0 resection shows good prognostic outcomes, with a 5-year survival rate $>50 \%(14,15)$. Retrospective studies have presented that the combination of hepatectomy and gastrectomy has visible survival outcome superiority (16-21). In the last two decades, along with the results of reported studies which demonstrated that radical surgery of primary gastric cancer and metastatic liver lesions had survival benefits, the Guidelines Committee of JGCA reconsidered the effect of surgical treatment in GCLM patients (22). Therefore, the role of LDT for GCLM is gradually being considered.

Previous therapeutic options for GCLM were HGCT, RFAG, TACEG, GCT and palliative chemotherapy (PCT). There are no randomized controlled clinical trials for GCLM therapies. In the

\footnotetext{
Abbreviations: GCLM, gastric cancer with liver metastasis; LDT, Liver-directed treatment; SUCRA, surface under the cumulative ranking curves; JGCA, Japan Gastric Cancer Association; NCCN, National Comprehensive Cancer Network; SCT, systemic chemotherapy; RoR, ratio of two odds ratios; IF, inconsistency factor; HGCT, hepatectomy and gastrectomy plus chemotherapy; GCT, gastrectomy plus chemotherapy; PCT, palliative chemotherapy; RFAG, radiofrequency ablation and gastrectomy plus chemotherapy; TACEG, transarterial chemoembolization and gastrectomy plus chemotherapy; HR, Hazard Ratio; NMA, network meta-analysis; OR, Odds ratio.
}

present literature, majority of the studies are retrospective studies, which were performed at a single center, with a limited number of patients. Although some studies have confirmed the superior therapeutic outcomes of LDT, the clinical pathological characteristics of the involved patients reveal some selection bias, therefore, their results are difficult to accept. We performed a network meta-analysis to evaluate the survival benefits of LDT and systemic chemotherapy in the treatment of GCLM.

\section{METHODS}

\section{Study Protocol}

This work was performed in accordance with the Preferred Reporting Items for Systematic Reviews and Meta-Analyses (PRISMA) of the Cochrane Handbook for Systematic Reviews of Intervention (23). The full protocol was registered and available on INPLASY (INPLASY2020120009).

\section{Search Strategy}

We retrieved literature published in between 1966 and September 1st, 2021 by searching PubMed, EMBASE, and Cochrane Library with the keywords (1) "stomach neoplasm" OR "gastric neoplasms" OR "cancer of stomach" OR "stomach cancers" OR "gastric cancer" AND (2) "liver metastases OR liver metastasis OR hepatic metastasis" AND (3) "operative surgical procedure" OR ablation OR liver resection OR hepatectomy OR gastrectomy OR chemotherapy OR "interventional therapy" and using the search strategies as illustrated in Supplementary Table 1. We selected and evaluated all relevant studies and review articles about GCLM and inquired the authors for unpublished raw data. Searches were limited to English-language publications. In addition, the reference lists of the retrieved articles were examined for potential eligible studies.

\section{Study Selection}

The inclusion criteria for the studies were: i. Systemic chemotherapy and surgical treatment; ii. Series of case control or cohort studies; iii. The number of patients were to be $>20$; iv. Consists of available endpoints, such as overall survival, 1-, 2-, 3-, and 5-year survival rates, median survival time, and postoperative complications. The exclusion criteria for the studies were: $i$. studies with insufficient data or no related endpoints; ii. Missing control group.

\section{Data Extraction}

Two researchers (MS and ZZ) independently extracted results from the enrolled articles in a standardized form. In addition, 
a third researcher (TL) was consulted in case there were disagreements. The information extracted from each study included the first author, country, year of publication, number of cases, treatment, sex, median or mean age of patients, study design, follow-up, median survival time. If a study did not report the Hazard Ratio (HR) of overall survival, we estimated HR and their corresponding 95\% confidence intervals (CIs) using the method described by Parmar et al. (24) and Tierney et al. (25). We recovered the data of Kaplan-Meier curves as recently described by us $(26,27)$.

\section{Quality Assessment}

We used the Newcastle-Ottawa Scale (NOS) to assess the quality of each included study. Scores $\geq 7$ were considered high quality. We used a "star system" for case-control studies (Supplementary Table 2).

\section{Publication Bias}

The funnel plots were used to establish publication bias. The funnel plot that was symmetrical near zero represented no publication bias.

\section{Statistical Analysis}

The primary endpoint of this network meta-analysis (NMA) was overall survival (OS), defined as the time from random assignment to date of death from any cause or date of last follow-up. Secondary endpoints were 1-, 2-, 3-year survival rates. A pair-wise meta-analysis was performed by STATA 13.0 (Stata Corp, College Station, TX). R-3.6.3 and R packages gemtc were applied to conduct the Bayesian NMA, and 95\% confidence intervals (CIs) were computed for HR in overall survival analysis. 1-, 2-, 3-year survival rates were analyzed while Odds Ratios (OR) with 95\% confidence intervals (CIs) was calculated by fixed-effects or random-effects model $(28,29)$. Z test was performed to evaluate the significance of overall effect size.
A network plot was then used to directly demonstrate the whole information of included studies (30). Depending on direct comparison and indirect comparison outcomes, we estimated the contribution of each direct treatment comparison in the whole network structure, which was presented in a contribution plot. The inconsistency factor (IF) was calculated to determine the possible inconsistency in network comparison. The 95\% CIs of IF values close to zero or the $\mathrm{p}$ value of $\mathrm{Z}$ test higher than 0.05 demonstrated there being no statistically significant inconsistency (31). Summary effects and corresponding predictive intervals were used to conclude relative mean effects and impact of heterogeneity in the network forest plot.

Finally, we calculated the surface under the cumulative ranking curve (SUCRA) of each treatment, which transformed the relative effects to the probability (2). SUCRA values range from 0 to $100 \%$. The treatment was more valuable if the SUCRA value was higher. According to the estimated probability value, the treatments were ranked, which showed the percentage of effectiveness a treatment achieves with reference to an imaginary ideal treatment. Small-study effects was adjusted by a model of network meta-regression, the variance of the log-odds ratios as covariation (32).

\section{RESULTS}

\section{Study Characteristics}

A total 6362 relevant articles were downloaded. The flow diagram documenting the search and inclusion of relevant studies is displayed in Figure 1. After considering the inclusion and exclusion criteria, a total of 23 retrospective studies involving 5472 GCLM patients were identified (12, $33-$ 57). At least one of the following treatments were assessed by the study: HGCT, RFAG, GCT, PCT, and TACEG. Eight studies were three-arm trials while fifteen studies were two-arm trials.

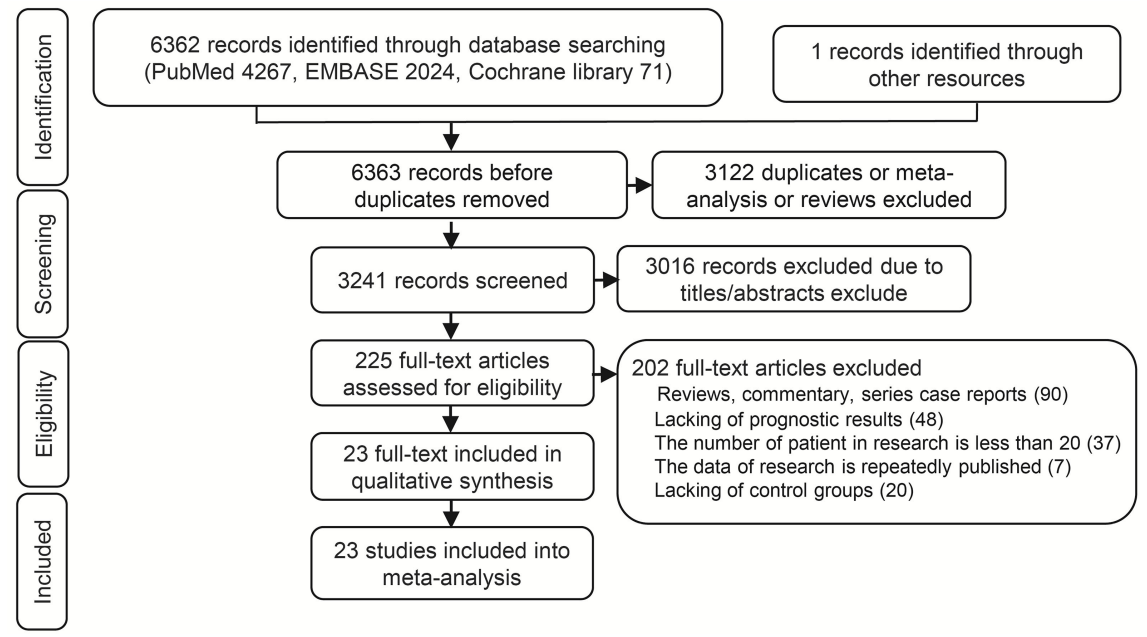

FIGURE 1 | Flow chart of the identification process for eligible studies. 
Further characteristics and Newcastle-Ottawa scale results regarding the included studies are presented in Table $\mathbf{1}$ (Supplementary Table 2).

\section{Direct Comparisons and Subgroup Analysis}

For OS and 1-, 2-, 3-year survival rate of all trials, meta-analysis of the direct comparisons showed significant better for HGCT treatments compared with GCT or PCT, with the exception of RFAG (Table 2). As to OS and 1-, 2-, 3-year survival rate of all trials, PCT predicted a significantly worse OS than GCT (Table 2). For 1-, 2-, 3-year survival rate, the results showed that RFAG indicated a better survival rate than GCT (Table 2). Analysis of Asian subgroups showed that HGCT were better than GCT in OS, and 1-, 2-, 3-year survival rate, RFAG were better than GCT in 1-, 2-, 3-year survival rate (Table 2). Overall, statistical heterogeneity was moderate, although for most comparisons 95\% CIs were wide and included values indicating very high or no heterogeneity, which portrayed the small number of studies available for every pair-wise comparison. In the meta-analyses of direct comparisons for OS and 1-, 2-, 3-year survival rate, $\mathrm{I}^{2}$ values higher than $40 \%$ were recorded for the comparisons HGCT versus GCT and HGCT versus PCT (Table 2).

\section{Network Meta-Analysis}

The network evidence plot is shown in Figure 2. Five treatments were included for analysis; HGCT, GCT, PCT, RFAG and TACEG, respectively. Comparing the studies with regards to their OS, 1-, 2-, 3-year survival rates, HGCT had the highest number of related studies and number of patients, while RFAG had the least number of patients and TACEG had the least number of related studies.

The contribution plot is presented in Figure 3. Ten comparisons were made in the network analysis. All of them are mixed comparisons. In the overall contribution of network analysis, the remarkable influence evidence in the comparisons of 1-, 2-, 3-year survival rate is PCT vs. TACEG (19.9\%), HGCT vs. RFAG (25.8\%), GCT vs. PCT (22.2\%), respectively.

There was no inconsistency between direct and indirect point estimates. In our network, there were 5 closed loops (Supplementary Figure 1). All confidence intervals for inconsistency factors (IFs) were compatible with zero inconsistency $(\mathrm{IF}=0)$ for all study outcomes (Supplementary Figure 1).

\section{Network Comparison}

The summary effects with 95\% CI are shown in Figure 4. In the comparison of the 5 treatments for 1-year survival rate, HGCT and RFAG were found to be more effective than GCT and PCT, respectively. GCT and TACEG was found to be more effective than PCT while there was no difference between HGCT and RFAG (Figure 4A). In the comparison of 2-year survival rates, HGCT and RFAG were found to be more effective than GCT and PCT, respectively. Other comparisons did not exhibit any significant differences (Figure 4B). In the comparison of 3-year survival rate, HGCT and RFAG were found to be more effective than GCT and PCT, respectively. GCT and TACEG was found to be more effective than PCT while there was no difference between HGCT and RFAG (Figure 4C).

\section{Ranking of Treatment}

Figures 4D-F shows the relative ranking distribution of estimated cumulative probabilities for each treatment. The surface under the cumulative ranking curve (SUCRA) was adjusted by small-study effects. The SUCRA value rankings of 1-year survival rate were HGCT (83.8\%), RFAG (81.5\%), TACEG (51.7\%), GCT (29.4\%), and PCT (3.7\%). The SUCRA value rankings of 2-year survival rate were RFAG (83\%), HGCT (68.7\%), TACEG (54\%), GCT (30.9\%), and PCT (13.4\%). The SUCRA value rankings of 3-year survival rate were RFAG (77\%), HGCT (72.9\%), TACEG (72.4\%), GCT (20.2\%), and PCT (7.5\%).

\section{Network Comparison, Ranking of Treatment and Subgroup Analysis of OS}

On OS analysis, four treatments (HGCT, GCT, RFAG, TACEG) showed an HR in favor of OS (HR range, 0.146-0.979) (Figure 5A) in Asian and Caucasian. RFAG was identified as the best option, based again on HR and the SUCRA (Figure 5B), followed by HGCT, TACEG, GCT and PCT. In Asian population, HGCT and RFAG had the most favorable HR (HGCT HR, 0.331 [95\% CI, 0.230-0.490; RFAG HR, 0.265 [95\% CI, 0.138-0.510]) (Figure 5C); they ranked, on median, first and second in all the simulations (Figure 5D).

\section{Subgroup Analysis of Network Comparison in Asian Population}

The summary effects in Asian population with 95\% CI are shown in Figure 6. In the comparison of the 5 treatments for 1-, 3- year survival rate, HGCT and RFAG were found to be more effective than GCT and PCT, respectively. TACEG was found to be more effective than PCT while there was no difference between GCT and PCT (Figures 6A, C). In the comparison of 2-year survival rates, HGCT and RFAG were found to be more effective than GCT and PCT, respectively. Other comparisons did not exhibit any significant differences (Figure 6B). The SUCRA value rankings of 1-year survival rate were HGCT, RFAG, TACEG, GCT, and PCT (Figure 6D). The SUCRA value rankings of 2-, 3year survival rate were RFAG, HGCT, TACEG, GCT, and PCT (Figures 6E, F).

\section{Publication Bias}

The funnel plot for network meta-analysis is presented in Figure 7. In general, all the selected studies were symmetrically distributed between the vertical line $(\mathrm{x}=0)$. Therefore, there was no noteworthy publication bias in our network meta-analysis.

\section{DISCUSSION}

In this network meta-analysis, we revealed that HGCT and RFAG and has remarkable survival benefits for GCLM patients when compared to TACEG, GCT and PCT. By OS and 2-, 3-year survival rate analysis, RFAG was identified as the best option, followed by HGCT, TACEG, GCT and PCT. By 1-year survival 
TABLE 1 | The major clinical and survival information of included eighteen studies.

\begin{tabular}{|c|c|c|c|c|c|c|c|c|c|c|c|c|c|c|c|c|c|c|c|}
\hline Study & Author & Country & Year & $\begin{array}{l}\text { Number } \\
\text { of } \\
\text { Patients }\end{array}$ & $\begin{array}{l}\text { Median age } \\
\text { (years) }\end{array}$ & $\begin{array}{l}\text { Follow up } \\
\text { (months) }\end{array}$ & $\begin{array}{l}\text { Hepatectomy } \\
\quad \text { arm }\end{array}$ & Synchronous & LVI & G3 & Unilobar & Solitary & T3-4 & Ro & $\mathrm{N}+$ & $\begin{array}{l}\text { Median } \\
\text { survival time } \\
\text { (months) }\end{array}$ & $\begin{array}{c}\text { Tumor size of } \\
\text { hepatic metastasis } \\
\text { (cm) }\end{array}$ & $\begin{array}{l}\text { Tumor size of } \\
\text { gastric cancer } \\
\text { (cm) }\end{array}$ & $\begin{array}{l}\text { NOS } \\
\text { score }\end{array}$ \\
\hline 1 & $\begin{array}{l}\text { Markar } \\
(37)\end{array}$ & Japan & 2016 & 217 & 65 & 8.3 & $\begin{array}{l}\text { Minor liver } \\
\text { resections }\end{array}$ & 78 & 63 & NA & 217 & NA & NA & NA & NA & NA & NA & NA & 9 \\
\hline 2 & Guner (12) & Japan & 2016 & 98 & NA & NA & NA & 39 & 49 & NA & 64 & NA & 72 & NA & 78 & HGCT 24; RFAG 23 & NA & NA & 7 \\
\hline 3 & Guan (33) & China & 2016 & 136 & 63 & NA & $\begin{array}{l}\text { Partial } \\
\text { hepatectomy }\end{array}$ & 71 & NA & NA & 62 & 136 & NA & NA & NA & $\begin{array}{l}\text { PCT 8.7; RFAG 10.1; } \\
\text { GCT 13.3 }\end{array}$ & NA & NA & 8 \\
\hline 4 & Yao (38) & China & 2015 & 49 & NA & NA & $\begin{array}{l}\text { Irregular } \\
\text { hepatectomy }\end{array}$ & NA & NA & NA & 34 & NA & NA & NA & NA & HGCT 24; GCT 12 & NA & NA & 8 \\
\hline 5 & $\begin{array}{l}\text { Shinohara } \\
\text { (39) }\end{array}$ & Japan & 2015 & 47 & 66.7 & NA & Liver resection & 28 & NA & 16 & 25 & 18 & 25 & NA & 41 & HGCT 22; GCT 7 & NA & NA & 8 \\
\hline 6 & $\begin{array}{l}\text { Ohkura } \\
(40)\end{array}$ & Japan & 2015 & 34 & 67.2 & 22.4 & NA & 34 & 29 & NA & NA & NA & 24 & NA & NA & NA & NA & NA & 8 \\
\hline 7 & $\operatorname{Liu}(41)$ & China & 2015 & 107 & $59 \pm 1.7$ & NA & NA & 107 & NA & 76 & 18 & NA & NA & NA & NA & $\begin{array}{l}\text { RFAG 5; } \\
\text { PCT } 3\end{array}$ & NA & NA & 8 \\
\hline 8 & Li (42) & China & 2015 & 49 & $61.4 \pm 9.5$ & 19.6 & NA & 49 & NA & 18 & NA & NA & 39 & NA & NA & GCT 20.5; PCT 9.1 & NA & NA & 9 \\
\hline 9 & Wang (58) & China & 2014 & 66 & 61 & 14 & $\begin{array}{l}\text { Radical } \\
\text { surgeries }\end{array}$ & 66 & NA & 6 & 34 & NA & 31 & NA & 33 & NA & NA & NA & 7 \\
\hline 10 & $\begin{array}{l}\text { Tiberio } \\
\text { (43) }\end{array}$ & Italy & 2014 & 195 & 68 & NA & Hepatectomy & NA & NA & NA & NA & NA & NA & 53 & NA & $\begin{array}{l}\text { HGCT 13; GCT 6.6; } \\
\text { РСT } 3\end{array}$ & NA & NA & 8 \\
\hline 11 & Chen (45) & China & 2013 & 114 & 54 & NA & $\begin{array}{l}\text { Major } \\
\text { hepatectomy }\end{array}$ & NA & NA & 23 & 63 & 51 & 78 & NA & 17 & HGCT 22.3; PCT 5.5 & NA & NA & 7 \\
\hline 12 & Miki (46) & Japan & 2012 & 50 & 70 & 33.4 & Hepatectomy & 41 & NA & NA & 25 & 20 & 40 & NA & NA & $\begin{array}{l}\text { HGCT 33.4; GCT } \\
\text { 10.5; PCT } 8.7\end{array}$ & NA & NA & 8 \\
\hline 13 & $\begin{array}{l}\text { Makino } \\
(47)\end{array}$ & Japan & 2010 & 63 & 65.8 & 16 & Hepatectomy & 31 & NA & NA & 30 & 24 & NA & NA & 54 & NA & NA & NA & 9 \\
\hline 14 & $\mathrm{Lu}(36)$ & China & 2010 & 60 & NA & NA & Hepatectomy & NA & NA & NA & 34 & 34 & NA & NA & NA & $\begin{array}{l}\text { HGCT 20; RFAG 18; } \\
\text { GCT } 16\end{array}$ & NA & NA & 7 \\
\hline 15 & Kim (36) & Korea & 2010 & 29 & 57.9 & 14.4 & NA & 12 & NA & 11 & 23 & NA & 21 & NA & NA & RFAG 30.7; GCT 6.8 & $\begin{array}{l}\text { RFAG } 2.8 \pm 1.4 ; \\
\text { GCT } 4.5 \pm 1.5\end{array}$ & $\begin{array}{l}\text { RFAG 5.1 } \pm 2.3 ; \\
\text { GCT 6.1 } 2.2 .2\end{array}$ & 7 \\
\hline 16 & $\begin{array}{l}\text { Cheon } \\
\text { (48) }\end{array}$ & Korea & 2008 & 58 & 61 & 15.5 & Hepatectomy & 42 & NA & 23 & 42 & 29 & NA & NA & 8 & $\begin{array}{l}\text { HGCT 21.7; RFAG 17; } \\
\text { GCT 8.1 }\end{array}$ & $\begin{array}{l}\text { HGCT } 2.4 \pm 1.7 \\
\text { RFAG } 2.1 \pm 1.4\end{array}$ & $\begin{array}{l}\text { HGCT } 5.7 \pm 2.4 \\
\text { RFAG } 6.6 \pm 3.1 \\
\text { GCT } 6.1 \pm 2.3\end{array}$ & 8 \\
\hline 17 & Li (35) & China & 2006 & 44 & NA & NA & Hepatectomy & NA & NA & 31 & NA & NA & NA & NA & NA & $\begin{array}{l}\text { HGCT 19.5; GCT 11; } \\
\text { PCT } 6.2\end{array}$ & NA & NA & 7 \\
\hline 18 & Li (49) & $\begin{array}{l}\text { China, } \\
\text { Taiwan }\end{array}$ & 2017 & 653 & $\begin{array}{c}68.28 \pm \\
12.87\end{array}$ & 33 & NA & NA & NA & NA & NA & NA & NA & NA & NA & $\begin{array}{l}\text { GCT 3.13; HGCT } \\
26.16\end{array}$ & NA & NA & 9 \\
\hline 19 & $\begin{array}{l}\text { Shirasu } \\
\text { (51) }\end{array}$ & Japan & 2018 & 24 & 64.6 & 47.9 & $\begin{array}{l}\text { Partial } \\
\text { hepatectomy }\end{array}$ & 16 & NA & 5 & 10 & 2 & NA & 9 & 1 & HGCT 24.8; PCT 38.1 & NA & NA & 9 \\
\hline 20 & Jagric (54) & Slovenia & 2020 & 42 & $65.2 \pm 8.49$ & NA & Metastasectomy & 42 & NA & 19 & 23 & 23 & 40 & 18 & NA & HGCT 9.3; GCT 4.2 & NA & NA & 9 \\
\hline 21 & $\begin{array}{l}\text { Picado } \\
(57)\end{array}$ & USA & 2018 & 3175 & 64 & $21(10-32)$ & NA & 42 & NA & 2168 & NA & NA & 260 & 137 & 1496 & GCT 16; PCT 9.7 & NA & NA & 9 \\
\hline 22 & Tang (55) & China & 2020 & 30 & 62 & 60 & NA & 35 & 18 & 33 & 36 & 31 & 46 & NA & 37 & $\begin{array}{l}\text { HGCT 21; RFAG 32; } \\
\text { GCT } 17\end{array}$ & $\begin{array}{l}\text { HGCT, } 2.9 \pm 1.6 \\
\text { RFAG, } 2.8 \pm 1.7 \\
\text { GCT, } 2.1 \pm 2.0\end{array}$ & NA & 9 \\
\hline 23 & Yu (56) & China & 2020 & 132 & $62.5(32-75)$ & $37.1(1-96)$ & NA & 132 & NA & 46 & 36 & NA & 121 & 39 & 111 & $\begin{array}{l}\text { HGCT 33.6(26.6- } \\
\text { 40.6); PCT 12.4(10.0- } \\
\text { 14.8) }\end{array}$ & NA & NA & 9 \\
\hline
\end{tabular}

NA, Not available; Synchronous, Number of patients with synchronous liver metastases; N+, Number of patients with lymph-node involvement of the primary cancer; T3-4, Number of patients with stage pT3 or pT4; LVI, Number of patients with lymphovascular involvement; G3, Number of patients with G3 primary cancer; Unilobar, Number of patients with unilobar liver involvement; RO, Number of patients who achieved an RO surgical removal on both primary cancer and liver metastases; Solitary, Number of patients with solitary liver metastases; HGCT, hepatectomy and gastrectomy plus chemotherapy; GCT, gastrectomy plus chemotherapy; PCT, palliative chemotherapy; RFAG, radiofrequency ablation and gastrectomy plus chemotherapy; TACEG, transarterial chemoembolization and gastrectomy plus chemotherapy. 
TABLE 2 | Summary estimates for 1-, 2-, 3-year survival rates in meta-analyses of direct comparisons between pairs of Liver-directed treatment and subgroup analysis of Asian for GCLM.

\begin{tabular}{|c|c|c|c|c|c|c|c|c|c|c|}
\hline Outcome & Subgroups & $\begin{array}{l}\text { No. of } \\
\text { trials }\end{array}$ & $\begin{array}{l}\text { OR, } \\
\text { FEM }\end{array}$ & $95 \% \mathrm{Cl}, \mathrm{FEM}$ & $\begin{array}{l}\text { P value of } \\
\text { FEM }\end{array}$ & $\begin{array}{l}\text { OR, } \\
\text { REM }\end{array}$ & 95\%Cl, REM & $\begin{array}{l}\text { P value of } \\
\text { REM }\end{array}$ & $\mathrm{I}^{2}$ & $\begin{array}{c}\text { Heterogeneity } \\
\text { P }\end{array}$ \\
\hline \multicolumn{11}{|l|}{ All trials } \\
\hline \multirow[t]{4}{*}{ OS } & GCT vs HGCT & 12 & 2.147 & $\begin{array}{l}\text { [1.819; } \\
2.534]\end{array}$ & $<0.0001$ & 2.209 & {$[1.744 ; 2.797]$} & $<0.0001$ & $41.30 \%$ & 0.066 \\
\hline & PCT vs HGCT & 8 & 2.797 & {$[2.3 ; 3.402]$} & $<0.0001$ & 2.664 & {$[1.991 ; 3.563]$} & $<0.0001$ & $47.30 \%$ & 0.065 \\
\hline & $\begin{array}{l}\text { RFAG vs } \\
\text { HGCT }\end{array}$ & 3 & 1.07 & $\begin{array}{r}{[0.725 ;} \\
1.580]\end{array}$ & 0.734 & 1.07 & {$[0.725 ; 1.580]$} & 0.734 & $0.00 \%$ & 0.81 \\
\hline & GCT vs PCT & 2 & 0.551 & $\begin{array}{c}{[0.467 ;} \\
0.650]\end{array}$ & $<0.0001$ & 0.551 & {$[0.467 ; 0.650]$} & $<0.0001$ & $0.00 \%$ & 0.418 \\
\hline \multirow[t]{8}{*}{$\begin{array}{l}\text { 1-year survival } \\
\text { rates }\end{array}$} & HGCT vs GCT & 13 & 4.173 & $\begin{array}{r}{[3.090} \\
5.635]\end{array}$ & $<0.0001$ & 4.438 & [2.852; 6.905] & $<0.0001$ & $43.79 \%$ & 0.0455 \\
\hline & HGCT vs PCT & 8 & 5.831 & $\begin{array}{l}{[3.957 ;} \\
8.591]\end{array}$ & $<0.0001$ & 5.765 & $\begin{array}{l}\text { [3.286; } \\
\text { 10.113] }\end{array}$ & $<0.0001$ & $44.09 \%$ & 0.0847 \\
\hline & $\begin{array}{l}\text { HGCT vs } \\
\text { RFAG }\end{array}$ & 3 & 1.084 & $\begin{array}{r}{[0.538} \\
2.186]\end{array}$ & 0.8207 & 1.091 & [0.540; 2.203] & 0.8075 & $0.00 \%$ & 0.6299 \\
\hline & $\begin{array}{l}\text { HGCT vs } \\
\text { TACEG }\end{array}$ & 1 & 0.816 & $\begin{array}{l}{[0.211 ;} \\
3.159]\end{array}$ & 0.7688 & 0.816 & {$[0.211 ; 3.159]$} & 0.7688 & NA & $<0.0001$ \\
\hline & GCT vs PCT & 7 & 2.957 & $\begin{array}{l}\text { [2.308; } \\
3.788]\end{array}$ & $<0.0001$ & 2.944 & {$[2.297 ; 3.775]$} & $<0.0001$ & $0.00 \%$ & 0.7182 \\
\hline & GCT vs RFAG & 3 & 0.248 & $\begin{array}{c}{[0.100} \\
0.617]\end{array}$ & 0.0027 & 0.25 & [0.099; 0.633] & 0.0035 & $0.00 \%$ & 0.5505 \\
\hline & GCT vs TACEG & 2 & 1.103 & $\begin{array}{l}{[0.559} \\
2.174]\end{array}$ & 0.7779 & 0.688 & {$[0.055 ; 8.540]$} & 0.7711 & $89.22 \%$ & 0.0023 \\
\hline & PCT vs TACEG & 2 & 0.34 & $\begin{array}{l}{[0.186} \\
0.622]\end{array}$ & 0.0005 & 0.322 & {$[0.086 ; 1.206]$} & 0.0925 & $78.12 \%$ & 0.0325 \\
\hline \multirow[t]{8}{*}{$\begin{array}{l}\text { 2-year survival } \\
\text { rates }\end{array}$} & HGCT vs GCT & 8 & 5.311 & $\begin{array}{l}\text { [3.353; } \\
8.410]\end{array}$ & $<0.0001$ & 5.076 & $\begin{array}{l}\text { [2.303; } \\
11.185]\end{array}$ & 0.0001 & $55.67 \%$ & 0.0271 \\
\hline & HGCT vs PCT & 6 & 4.707 & $\begin{array}{l}\text { [2.673; } \\
8.289]\end{array}$ & $<0.0001$ & 4.824 & $\begin{array}{l}\text { [1.270; } \\
18.330]\end{array}$ & 0.0209 & $70.83 \%$ & 0.0042 \\
\hline & $\begin{array}{l}\text { HGCT vs } \\
\text { RFAG }\end{array}$ & 2 & 0.725 & $\begin{array}{l}{[0.296} \\
1.774]\end{array}$ & 0.4809 & 0.725 & {$[0.296 ; 1.775]$} & 0.481 & $0.00 \%$ & 0.7939 \\
\hline & $\begin{array}{l}\text { HGCT vs } \\
\text { TACEG }\end{array}$ & 1 & 0.468 & $\begin{array}{r}{[0.136} \\
1.611]\end{array}$ & 0.2284 & 0.468 & {$[0.136 ; 1.611]$} & 0.2284 & NA & 1 \\
\hline & GCT vs PCT & 5 & 3.106 & $\begin{array}{l}{[2.292 ;} \\
4.209]\end{array}$ & $<0.0001$ & 3.059 & {$[2.260 ; 4.140]$} & $<0.0001$ & $0.00 \%$ & 0.9282 \\
\hline & GCT vs RFAG & 3 & 0.161 & $\begin{array}{c}{[0.050 ;} \\
0.513]\end{array}$ & 0.002 & 0.158 & [0.026; 0.958] & 0.0448 & $39.87 \%$ & 0.1895 \\
\hline & GCT vs TACEG & 2 & 0.702 & $\begin{array}{r}{[0.291} \\
1.691]\end{array}$ & 0.4303 & 0.488 & $\begin{array}{c}{[0.001 ;} \\
238.526]\end{array}$ & 0.8202 & $91.40 \%$ & 0.0006 \\
\hline & PCT vs TACEG & 2 & 0.849 & $\begin{array}{l}{[0.238} \\
3.028]\end{array}$ & 0.801 & 0.732 & $\begin{array}{l}\text { [0.025; } \\
\text { 21.063] }\end{array}$ & 0.8555 & $77.22 \%$ & 0.0361 \\
\hline \multirow[t]{8}{*}{$\begin{array}{l}\text { 3-year survival } \\
\text { rates }\end{array}$} & HGCT vs GCT & 11 & 4.742 & $\begin{array}{l}\text { [2.699; } \\
8.333]\end{array}$ & $<0.0001$ & 4.556 & {$[2.574 ; 8.061]$} & $<0.0001$ & $0.00 \%$ & 0.9186 \\
\hline & HGCT vs PCT & 7 & 5.157 & $\begin{array}{l}\text { [2.628; } \\
10.120]\end{array}$ & $<0.0001$ & 5.565 & $\begin{array}{l}\text { [1.811; } \\
17.103]\end{array}$ & 0.0027 & $48.73 \%$ & 0.0689 \\
\hline & $\begin{array}{l}\text { HGCT vs } \\
\text { RFAG }\end{array}$ & 3 & 0.877 & $\begin{array}{c}{[0.454} \\
1.695]\end{array}$ & 0.6967 & 0.877 & {$[0.453 ; 1.698]$} & 0.696 & $0.00 \%$ & 0.7758 \\
\hline & $\begin{array}{l}\text { HGCT vs } \\
\text { TACEG }\end{array}$ & 1 & 0.988 & $\begin{array}{l}{[0.248} \\
3.935]\end{array}$ & 0.9866 & 0.988 & [0.248; 3.935] & 0.9866 & NA & 1 \\
\hline & GCT vs PCT & 5 & 4.227 & $\begin{array}{l}{[2.822 ;} \\
6.332]\end{array}$ & $<0.0001$ & 4.295 & {$[2.908 ; 6.345]$} & $<0.0001$ & $0.00 \%$ & 0.9903 \\
\hline & GCT vs RFAG & 3 & 0.153 & $\begin{array}{c}{[0.034 ;} \\
0.687]\end{array}$ & 0.0144 & 0.176 & [0.037; 0.842] & 0.0296 & $0.00 \%$ & 0.5427 \\
\hline & GCT vs TACEG & 2 & 0.332 & $\begin{array}{r}{[0.067 ;} \\
1.645]\end{array}$ & 0.1768 & 0.454 & $\begin{array}{l}\text { [0.011; } \\
\text { 19.365] }\end{array}$ & 0.6799 & $65.83 \%$ & 0.0871 \\
\hline & PCT vs TACEG & 1 & 0.139 & $\begin{array}{l}\text { [0.006; } \\
\text { 3.507] }\end{array}$ & 0.2309 & 0.139 & [0.006; 3.507] & 0.2309 & NA & $<0.0001$ \\
\hline \multicolumn{11}{|c|}{ Trials of Asian population } \\
\hline \multirow[t]{2}{*}{ OS } & GCT vs HGCT & 10 & 2.283 & $\begin{array}{l}{[1.861 ;} \\
2.800]\end{array}$ & $<0.0001$ & 2.308 & {$[1.695 ; 3.143]$} & $<0.0001$ & $47.40 \%$ & 0.047 \\
\hline & PCT vs HGCT & 7 & 2.67 & $\begin{array}{l}{[2.142 ;} \\
3.328]\end{array}$ & $<0.0001$ & 2.505 & {$[1.770 ; 3.546]$} & $<0.0001$ & $51.90 \%$ & 0.052 \\
\hline
\end{tabular}


TABLE 2 | Continued

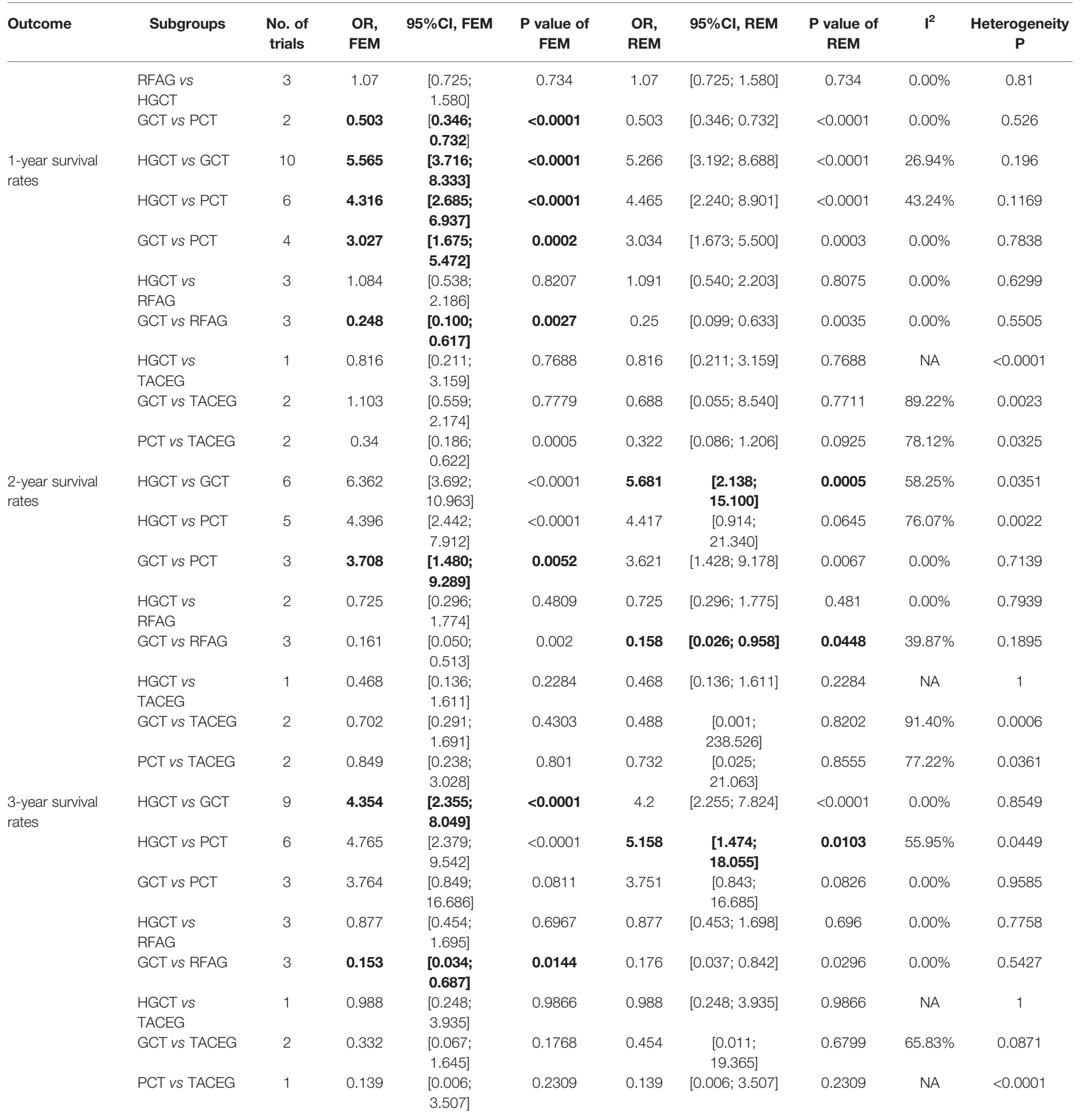

OR, Odds ratios; Cl, confidence interval; FEM, Fixed-effect Model; REM, Random-effect Model; NA, not available.

$P^{2}$ : index for assessing heterogeneity; value $\geq 40 \%$ indicates a moderate to high heterogeneity.

Bold indicate statistically significant values $(P<0.05)$.

rate analysis, HGCT and RFAG were identified as the most effective options. Due to non-specific symptoms, majority of gastric cancer patients were initially diagnosed with distant metastases. GCLM was considered as IV stage. The NCCN guideline recommends systemic chemotherapy as the standard cure for this group of patients. However, some controversies have been reported in the past two decades. Kim et al. (59) reported that gastrectomy or gastrectomy plus hepatectomy in GCLM patients has survival benefits when compared to chemotherapy. Tsujimoto et al. (60) showed that the 5-year survival rate of GCLM patients after hepatic resection was $31.5 \%$, median survival time was 34 months. They also found that 


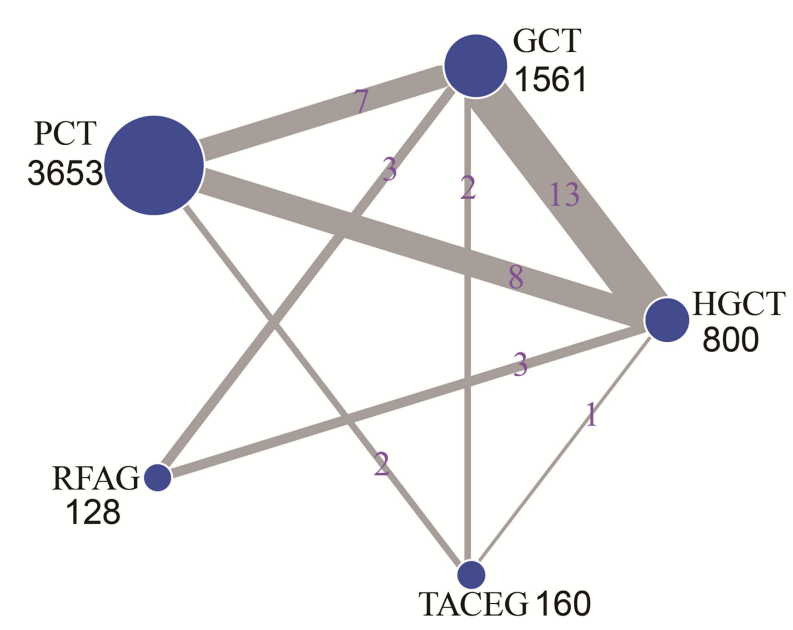

FIGURE 2 | Network of eligible comparisons for the multiple-treatment meta-analysis. The size of each node represents the total sample size of treatment. The thickness of each line represents the total number of studies that compare each other. HGCT, hepatectomy and gastrectomy plus chemotherapy; GCT, gastrectomy plus chemotherapy; PCT, palliative chemotherapy; RFAG, radiofrequency ablation and gastrectomy plus chemotherapy; TACEG, transarterial chemoembolization and gastrectomy plus chemotherapy.

gastric tumor less than $6 \mathrm{~cm}$ and D2 lymphadenectomy were important factors for prognosis. Song's study (61) suggested that surgical hepatic resection is beneficial for long-term survival in selected patients, with a 3-year survival rate of $47.6 \%$. Groundbreaking by survival benefits of combined conversion therapy with surgery in patients with colorectal cancer liver metastases, numerous general surgeons navigated HGCT or RFAG in GCLM, which was thought over as a crucial strategy to alleviation disease and to prolong patient life (62-79). Liverdirected treatment (LDT) options for GCLM patients and surgical treatments were gradually attempted (12). If complete resection of liver metastases is possible, considering adequate hepatic reserve and surgical security, radical operations for primary gastric cancer and liver metastases lesions should be attempted $(22,79)$.
Considering the retrospective nature of the included studies and different selection biases for choosing patients on whom to perform radical surgery, their outcomes can hardly be regarded as a rationale in the treatment of GCLM, but it broadens the horizon of radical surgery in the selected GCLM patients. Furthermore, its prognostic value is considerable. Hepatic resection for liver metastases from colorectal cancer has been recommended as a standard treatment, 5-year survival was almost $40 \%(80)$. When the number of liver metastasis tumor $\leq$ 3 , the diameter of single metastasis lesion $\leq 3 \mathrm{~cm}$, the resection of primary gastric cancer and liver metastasis can also offer survival benefits in the GCLM patient (40). The security of surgical treatments for GCLM patients has also been confirmed. It does not enhance postoperative mortality (37). Studies also reported that GCLM patients with hepatectomy and

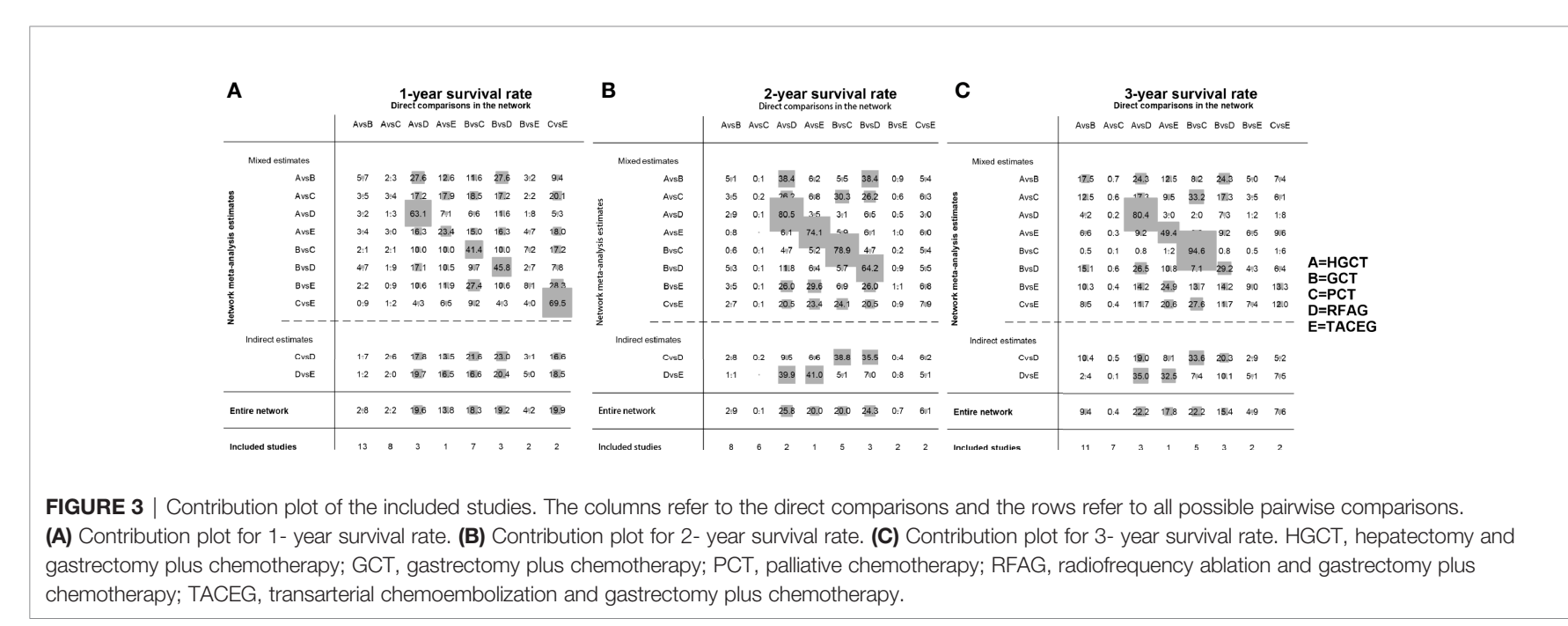


A

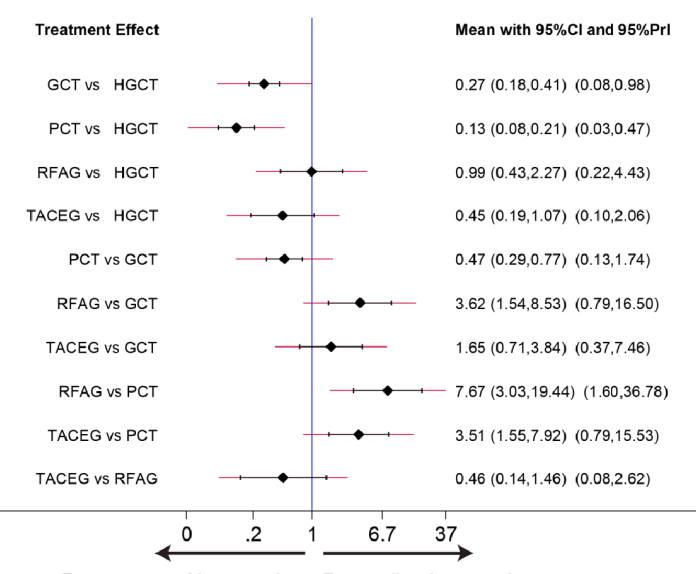

Favors second intervention Favors first intervention

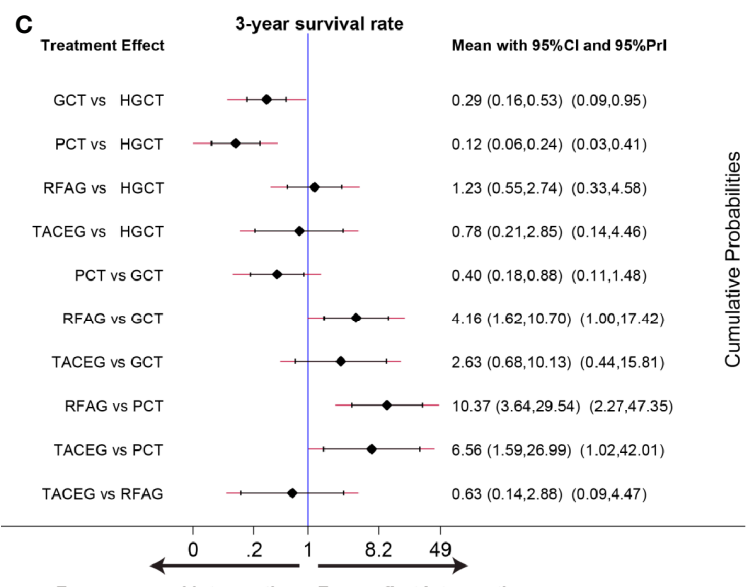

Favors second intervention Favors first intervention 2-year survival rate

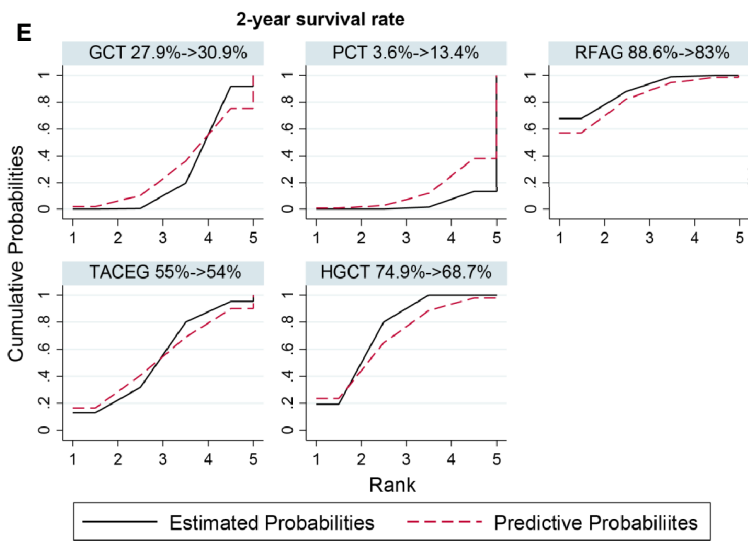

B

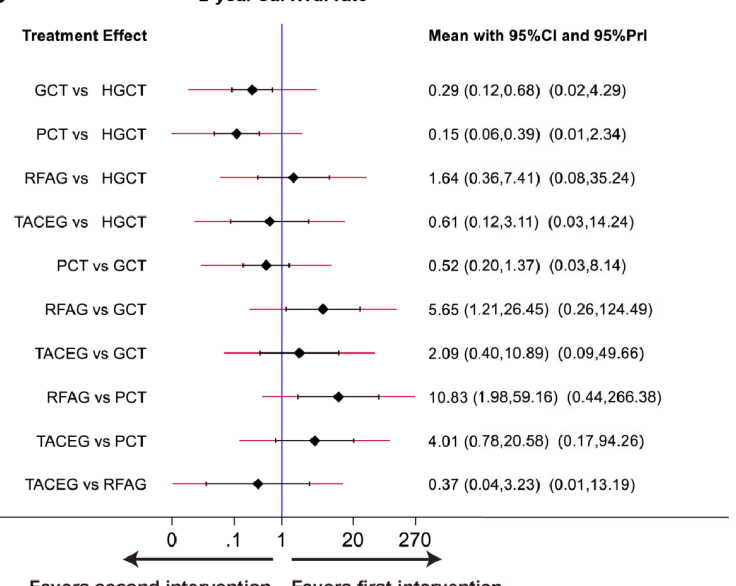

Favors second intervention Favors first intervention
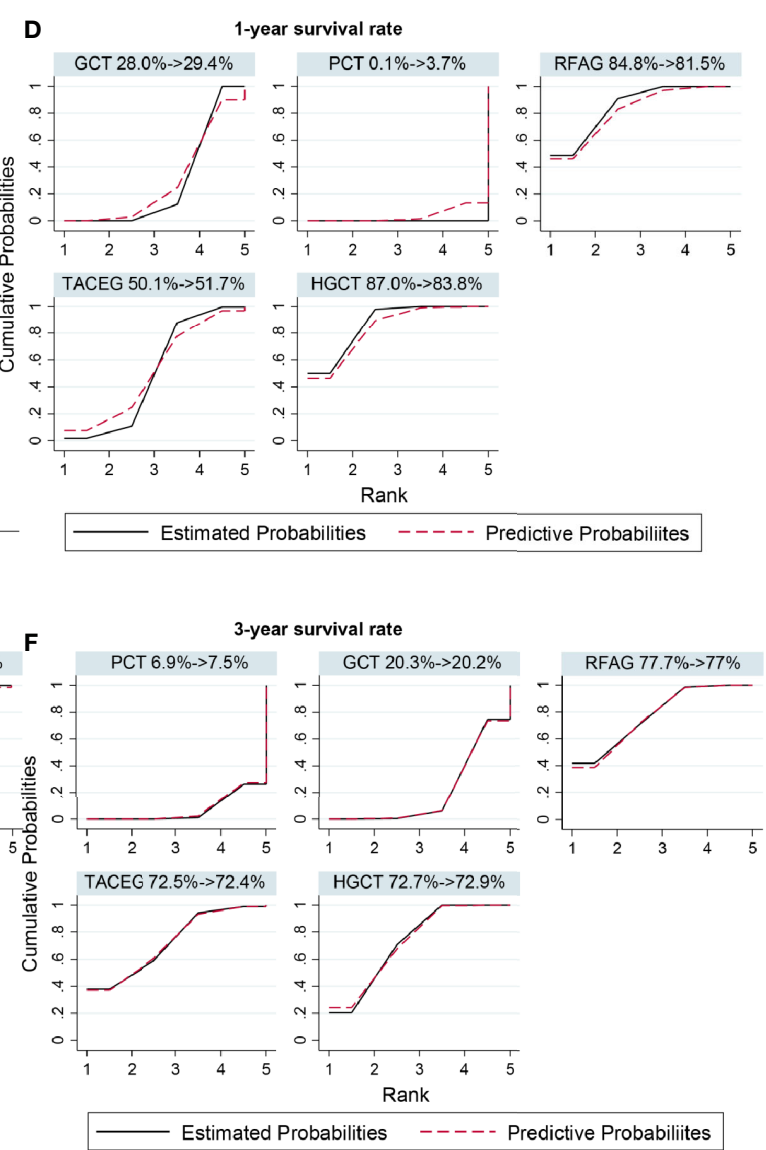

FIGURE 4 | Confidence interval plot and SUCRA for the network analysis. The black and red solid lines represent the 95\% confidence interval and the predictive intervals for summary relative risks for each comparison in the confidence interval plot. The blue line is the line of no effect (relative risk equal to 1). Confidence interval plot of 1-, 2- and 3- year survival rate (A-C). SUCRA of 1-, 2- and 3- year survival rate (D-F). Black solid lines correspond to the unadjusted model and red dashed lines to the adjusted for small effects model. Ranking indicates the probability to be the best treatment.

gastrectomy exhibited favorable prognosis (2, 81-85). Therefore, we have confidence in the survival benefits of surgical hepatic resection. The value of this surgical treatment option is worth considering. According to the SUCRA values of the NMA, HGCT exhibited remarkable 1-, 2-, or 3-year survival and OS outcomes. Hepatic resection has survival superiority for selected GCLM patients.

RFAG was superior to the other therapies in 2-, or 3-year survival rate and OS analysis in this NMA. RFA has been thought over a less invasive therapeutic choice for GCLM (86). RFA can 

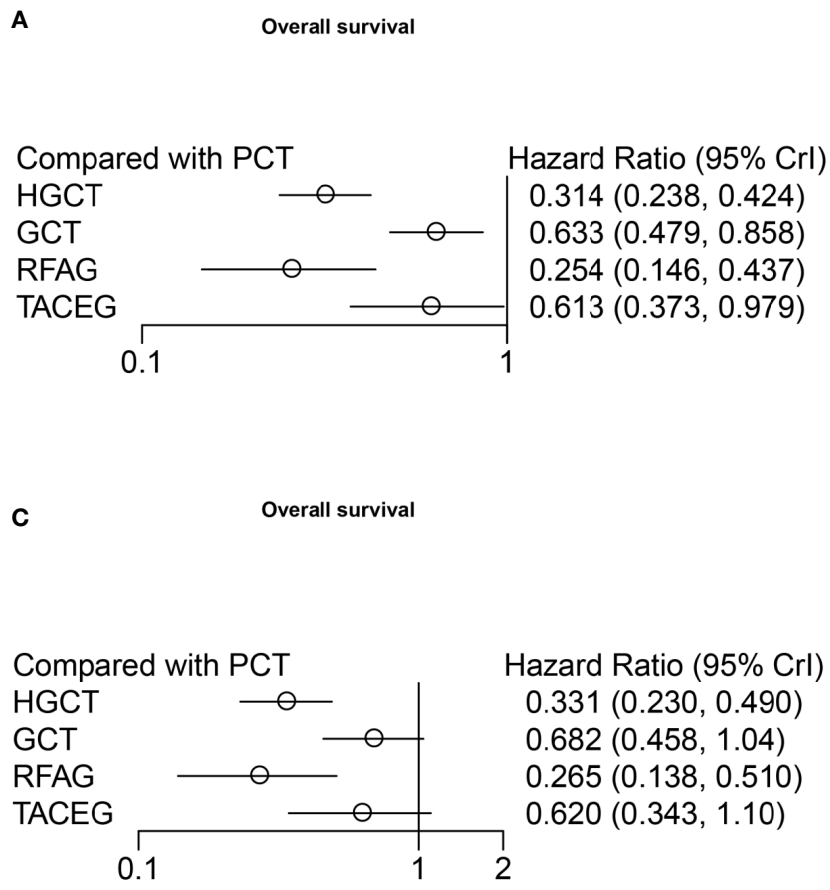
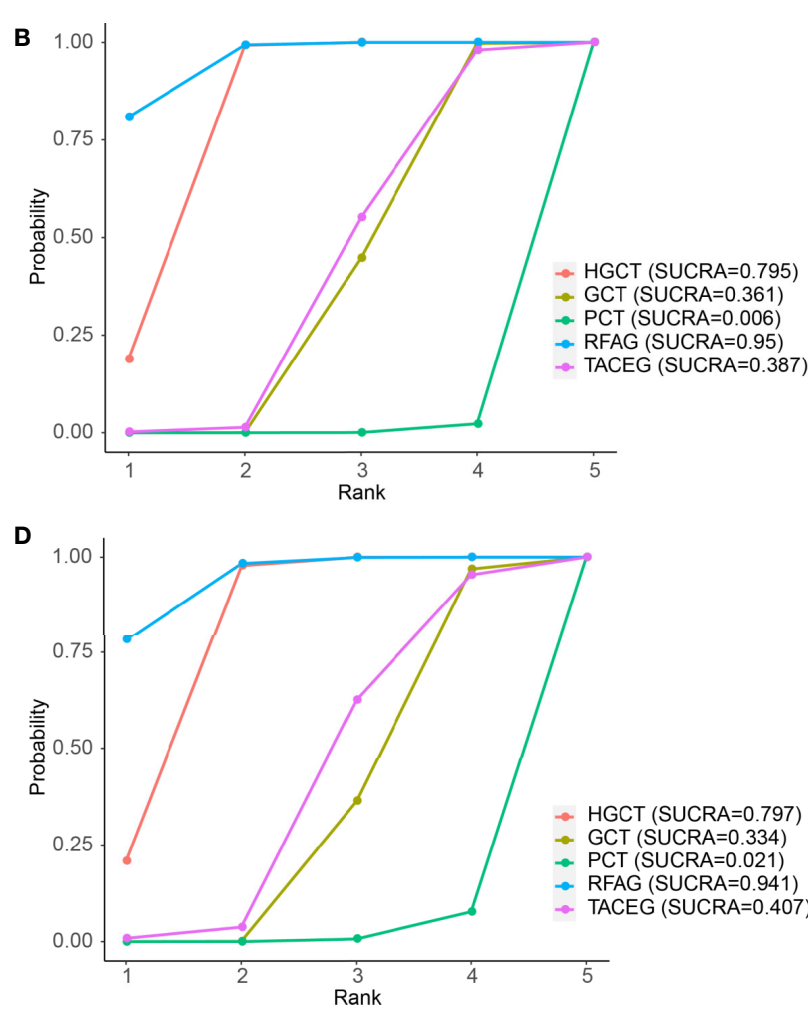

FIGURE 5 | Results from network meta-analyses incorporating direct and indirect comparisons between the eligible interventions (OS). (A) Forest plot of each intervention versus PCT. (B) SUCRA plot of each intervention. (C) Forest plot of each intervention versus PCT in the Asian population. (D) SUCRA plot of each intervention in the Asian population.

be used combined with systemic treatments (chemotherapy, targeted treatment, and immunotherapy), surgeries, and radiotherapy. RFAG which was radiofrequency ablation and gastrectomy plus chemotherapy showed comparable outcomes to curative resection $(12,48,87)$. Cheon's study suggested that a survival benefit of RFAG with curative intent was observed as compared with GCT, as evidenced by an improvement of $20.8 \%$ in the 5-year survival rate, corresponding to a $64.0 \%$ reduction in the risk of death (48). Kim et al. reported that The RFAG group showed a $76 \%$ decreased death rate compared to the GCT, was received well, and was found to be minor complications (34). Guner et al. suggested that in select patients with GCLM, HGCT and RFA showed satisfactory and comparable short- and longterm results, possible liver-directed treatment options for GCLM patients should be considered on an individual basis (12). Tang et al. suggested that OS were satisfactory and comparable between RFA and HGCT but better than those of chemotherapy, RFA is an appropriate option for patients with gastric cancer who have a solitary liver metastasis measuring $\leq 3.0 \mathrm{~cm}$ (55).

In our network analysis, we adopted several methods to control potential bias. First, the quality of all included studies was assessed by the Newcastle-Ottawa scale. The contribution plot was then performed to seek for significant bias in the network analysis. HGCT and RFAG exhibited the most impact on the 1-, 2- and 3-year survival rates, with $19.6 \%, 25.8 \%$ and
$22.2 \%$ respectively, which was attributed to the small number of included patients. We also applied the small-study effects to adjust the value of SUCRA to control for potential bias. There was a low risk of publication bias.

Our study had some limitations. The retrospective nature of the included studies enhances the possibility of selection bias between different centers. Patient characteristics such as the number and size of hepatic metastasis, the location of metastasis lesions, the postoperative supportive treatment and adjuvant chemotherapy, which are vital prognostic factors to influence the survival benefits in GCLM patients could hardly ensure balance. However, it is difficult to perform prospective cohort studies for this group of patients die to the small number of GCLM patients in single centers and dismal prognosis with systemic chemotherapy. Our results recommend the HGCT or RFAG treatment option for GCLM patients when resection of gastric cancer and liver metastases lesions is feasible. This recommendation is in tandem with those of the EORTC and JCOG studies. Liver resection or RFA is a favorable option for GCLM patients without extrahepatic metastases, peritoneal dissemination and multiple hepatic metastases (22). Meanwhile, the maximum liver metastatic tumor size for which RFA is safe and effective remains highly controversial $(55,88)$.

To sum up, HGCT was found to exhibit superior therapeutic effects for GCLM patients while RFAG was found to be a prospective therapeutic alternative. Although we obtained data 
A

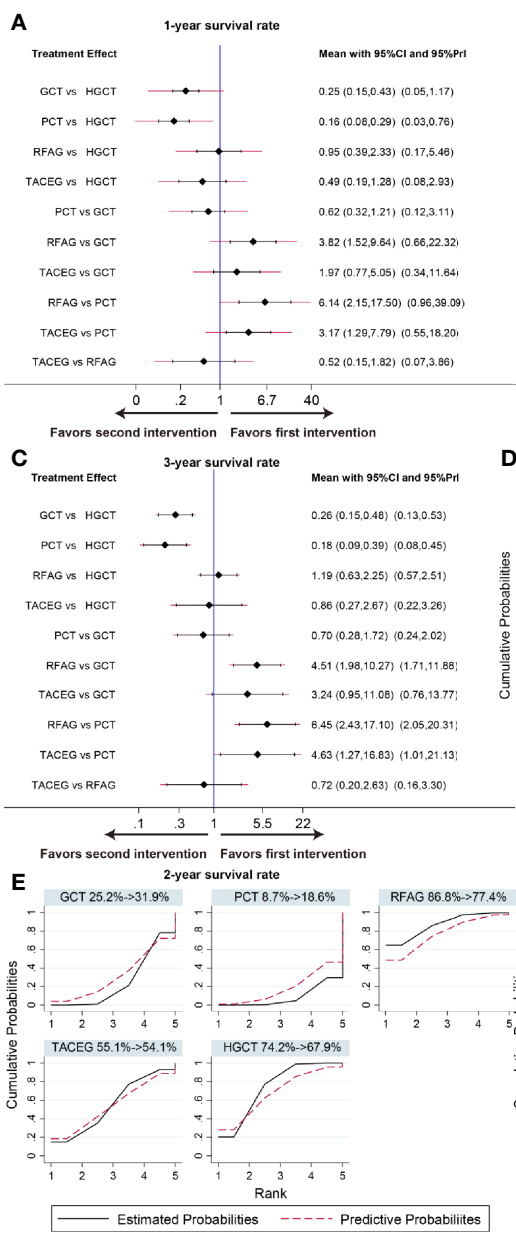

B

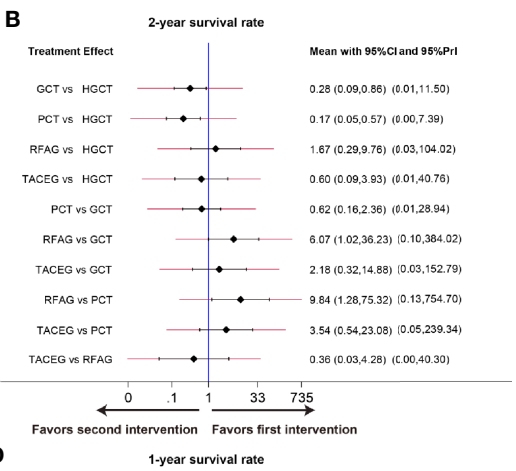

1-year survival rate

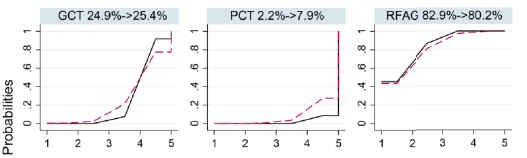

TACEG 53.6\% $\rightarrow 53.6 \%$ HGCT 86.5\% $282.9 \%$

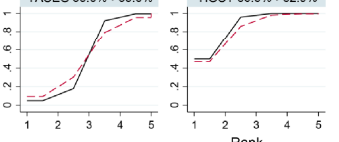

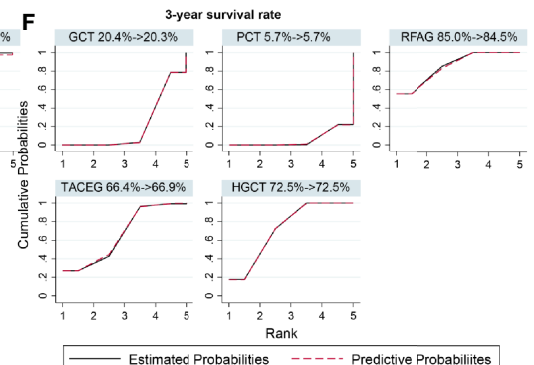

FIGURE 6 | Confidence interval plot and SUCRA for the network analysis in the Asian population. The black and red solid lines represent the 95\% confidence interval and the predictive intervals for summary relative risks for each comparison in the confidence interval plot. The blue line is the line of no effect (relative risk equal to 1). Confidence interval plot of 1-, 2- and 3- year survival rate in the Asian population (A-C). SUCRA of 1-, 2- and 3- year survival rate (D-F) in the Asian population. Black solid lines correspond to the unadjusted model and red dashed lines to the adjusted for small effects model. Ranking indicates the probability to be the best treatment.
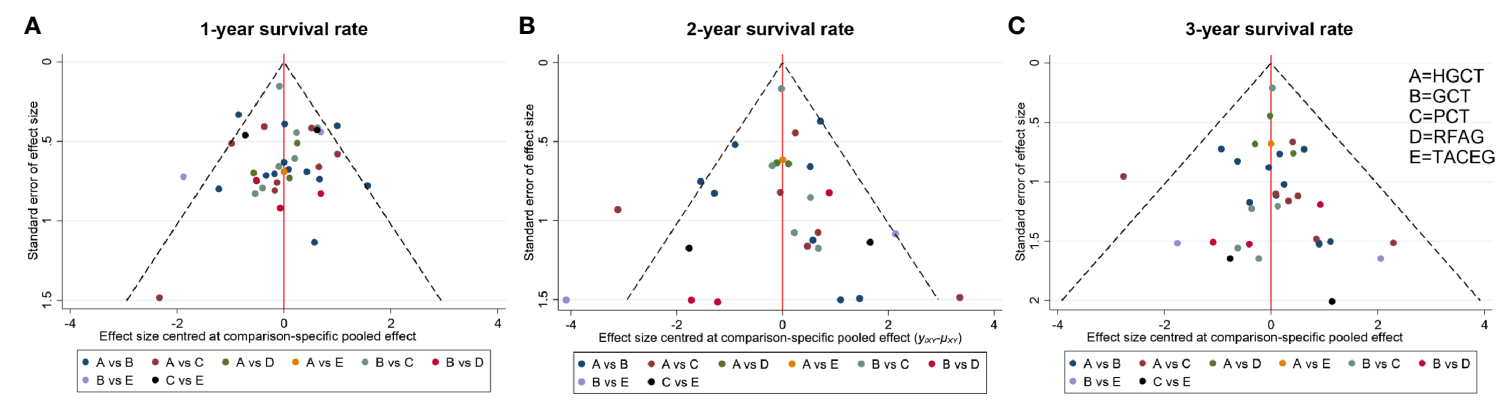

FIGURE 7 | Comparison-adjusted funnel plot for the network meta-analysis. The red line suggests the null hypothesis that the study-specific effect sizes do not differ from the respective comparison-specific pooled effect estimates. Different colors represent different comparisons. (A) 1- year survival rate. (B) 2- year survival rate. (C) 3- year survival rate. 
from retrospective studies, we confirmed the role of RFAG and HGCT as a therapeutic option for GCLM. Large-scale prospective studies in multiple centers are needed to further evaluate the survival benefits of potential radical surgery or RFAG in selected patients.

\section{DATA AVAILABILITY STATEMENT}

The original contributions presented in the study are included in the article/Supplementary Material. Further inquiries can be directed to the corresponding authors.

\section{AUTHOR CONTRIBUTIONS}

Conceptualization: TL and MS. Data curation: MS, LX, and ZZ. Formal analysis: SW and HD. Funding acquisition: XG, LX, and MS. Methodology: TL and MS. Project administration: TL and HD. Resources: MS and HD. Software: MS, ZZ, and SW. Supervision: TL and HD. Validation: TL. Visualization: MS and LX. Writing - original draft: MS, ZZ, and TL. Writing review \& editing: HD and TL. All authors contributed to the article and approved the submitted version.

\section{FUNDING}

This research was supported by the National Natural Science Foundation of China (81902498), Hubei Provincial Natural

\section{REFERENCES}

1. Varon C, Azzi-Martin L, Khalid S, Seeneevassen L, Menard A, Spuul P. Helicobacters and Cancer, Not Only Gastric Cancer? Semin Cancer Biol (2021). doi: 10.1016/j.semcancer.2021.08.007

2. Mbuagbaw L, Rochwerg B, Jaeschke R, Heels-Andsell D, Alhazzani W, Thabane L, et al. Approaches to Interpreting and Choosing the Best Treatments in Network Meta-Analyses. Syst Rev (2017) 6(1):79. doi: 10.1186/s13643-017-0473-z

3. Navashenaq JG, Shabgah AG, Banach M, Jamialahmadi T, Penson PE, Johnston TP, et al. The Interaction of Helicobacter Pylori With Cancer Immunomodulatory Stromal Cells: New Insight Into Gastric Cancer Pathogenesis. Semin Cancer Biol (2021). doi: 10.1016/j.semcancer.2021.09.014

4. Kulik U, Lehner F, Bektas H, Klempnauer J. Liver Resection for Non-Colorectal Liver Metastases - Standards and Extended Indications. Viszeralmedizin: Gastrointest Med Surg (2015) 31(6):394-8. doi: 10.1159/000439419

5. Page AJ, Weiss MJ, Pawlik TM. Surgical Management of Noncolorectal Cancer Liver Metastases. Cancer (2014) 120(20):3111-21. doi: 10.1002/ cncr. 28743

6. pt?>Riddiough GE, Jalal Q, Perini MV, Majeed AW. Liver Regeneration and Liver Metastasis. Semin Cancer Biol (2021) 71:86-97. doi: 10.1016/ j.semcancer.2020.05.012

7. Ma B, Wells A, Wei L, Zheng J. Prostate Cancer Liver Metastasis: Dormancy and Resistance to Therapy. Semin Cancer Biol (2021) 71:2-9. doi: 10.1016/ j.semcancer.2020.07.004

8. Wang H, Li X, Peng R, Wang Y, Wang J. Stereotactic Ablative Radiotherapy for Colorectal Cancer Liver Metastasis. Semin Cancer Biol (2021) 71:21-32. doi: 10.1016/j.semcancer.2020.06.018

9. Tiberio GAM, Roviello F, Donini A, De Manzoni G. Hepatic Metastases From Gastric Cancer: A Surgical Perspective. World J Gastroenterol (2015) 21 (41):11489-92. doi: 10.3748/wjg.v21.i41.11489
Science Foundation (2019CFB450, 2019CFB177, 2016CFB530), Natural Science Foundation of Hubei Provincial Department of Education (Q20182105), Chen Xiao-ping Foundation for the Development of Science and Technology of Hubei Provincial (CXPJJH11800001-2018333), The Scientific and Technological Project of Shiyan City of Hubei Province (18Y35), The Foundation of Health and Family Planning Commission of Hubei Province (WJ2021Q007), and Innovation and Entrepreneurship Training Program (201810929005, 201810929009, 201810929068, 201813249010, S201910929009, S201910929045, S202013249005, S202013249008 and 202010929009).

\section{ACKNOWLEDGMENTS}

We thanked Home for Researchers editorial team (www.homefor-researchers.com) for language editing service.

\section{SUPPLEMENTARY MATERIAL}

The Supplementary Material for this article can be found online at: https://www.frontiersin.org/articles/10.3389/fonc.2021.675870/ full\#supplementary-material

Supplementary Figure 1 | Inconsistency plot for the network meta-analysis. (A) inconsistency plot of 1- year survival rate. (B) inconsistency plot of 2- year survival rate. (C) inconsistency plot of 3- year survival rate.

10. Romano F, Garancini M, Uggeri F, Degrate L, Nespoli L, Gianotti L, et al. Surgical Treatment of Liver Metastases of Gastric Cancer: State of the Art. World J Surg Oncol (2012) 10:157. doi: 10.1186/1477-7819-10-157

11. Guner A, Yildirim R. Surgical Management of Metastatic Gastric Cancer: Moving Beyond the Guidelines. Transl Gastroenterol Hepatol (2019) 4:58. doi: 10.21037/tgh.2019.08.03

12. Guner A, Son T, Cho I, Kwon IG, An JY, Kim HI, et al. Liver-Directed Treatments for Liver Metastasis From Gastric Adenocarcinoma: Comparison Between Liver Resection and Radiofrequency Ablation. Gastric Cancer (2016) 19(3):951-60. doi: 10.1007/s10120-015-0522-Z

13. Melichar B, Vobořil Z, Cerman J, Melicharová K, Nožička J, Mergancová J, et al. Hepatic Arterial Infusion Chemotherapy in Gastric Cancer: A Report of Four Cases and Analysis of the Literature. Tumori (2004) 90(4):428-34. doi: 10.1177/030089160409000414

14. Jeong S, Heo JS, Park JY, Choi DW, Choi SH. Surgical Resection of Synchronous and Metachronous Lung and Liver Metastases of Colorectal Cancers. Ann Surg Treat Res (2017) 92(2):82-9. doi: 10.4174/astr.2017.92.2.82

15. Hosokawa I, Allard MA, Gelli M, Ciacio O, Vibert E, Cherqui D, et al. LongTerm Survival Benefit and Potential for Cure After R1 Resection for Colorectal Liver Metastases. Ann Surg Oncol (2016) 23(6):1897-905. doi: 10.1245/s10434-015-5060-8

16. Saito A, Korenaga D, Sakaguchi Y, Ohno S, Ichiyoshi Y, Sugimachi K. Surgical Treatment for Gastric Carcinomas With Concomitant Hepatic Metastasis. Hepato-Gastroenterology (1996) 43(9):560-4.

17. Fujii K, Fujioka S, Kato K, Machiki Y, Kutsuna Y, Ishikawa A, et al. Resection of Liver Metastasis From Gastric Adenocarcinoma. Hepato-Gastroenterology (2001) 48(38):368-71.

18. Sakamoto Y, Ohyama S, Yamamoto J, Yamada K, Seki M, Ohta KI, et al. Surgical Resection of Liver Metastases of Gastric Cancer: An Analysis of a 17Year Experience With 22 Patients. Surgery (2003) 133(5):507-11. doi: $10.1067 / \mathrm{msy} .2003 .147$ 
19. Roh HR, Suh KS, Lee HJ, Yang HK, Choe KJ, Lee KU. Outcome of Hepatic Resection for Metastatic Gastric Cancer. Am Surg (2005) 71(2):95-9. doi: $10.1177 / 000313480507100201$

20. Morise Z, Sugioka A, Hoshimoto S, Kato T, Ikeda M, Uyama I, et al. The Role of Hepatectomy for Patients With Liver Metastases of Gastric Cancer. Hepatogastroenterology (2008) 55(85):1238-41.

21. Thelen A, Jonas S, Benckert C, Lopez-Hänninen E, Neumann U, Rudolph B, et al. Liver Resection for Metastatic Gastric Cancer. Eur J Surg Oncol (2008) 34 (12):1328-34. doi: 10.1016/j.ejso.2008.01.022

22. Kataoka K, Kinoshita T, Moehler M, Mauer M, Shitara K, Wagner AD, et al. Current Management of Liver Metastases From Gastric Cancer: What is Common Practice? New Challenge of EORTC and JCOG. Gastric Cancer (2017) 20:1-9. doi: 10.1007/s10120-017-0696-7

23. Moher D, Liberati A, Tetzlaff J, Altman DG, Group P. Preferred Reporting Items for Systematic Reviews and Meta-Analyses: The PRISMA Statement. Bmj (2009) 339:b2535. doi: 10.1136/bmj.b2535

24. Parmar MK, Torri V, Stewart L. Extracting Summary Statistics to Perform Meta-Analyses of the Published Literature for Survival Endpoints. Stat Med (1998) 17(24):2815-34. doi: 10.1002/(SICI)1097-0258(19981230)17:24<2815:: AID-SIM1 10>3.0.CO;2-8

25. Tierney JF, Stewart LA, Ghersi D, Burdett S, Sydes MR. Practical Methods for Incorporating Summary Time-to-Event Data Into Meta-Analysis. Trials (2007) 8:16. doi: 10.1186/1745-6215-8-16

26. Li Y, Du M, Wang S, Zha J, Lei P, Wang X, et al. Clinicopathological Implication of Long Non-Coding RNAs SOX2 Overlapping Transcript and Its Potential Target Gene Network in Various Cancers. Front Genet (2019) 10:1375. doi: $10.3389 /$ fgene.2019.01375

27. Sun M, Du M, Zhang W, Xiong S, Gong X, Lei P, et al. Survival and Clinicopathological Significance of SIRT1 Expression in Cancers: A MetaAnalysis. Front Endocrinol (2019) 10:121. doi: 10.3389/fendo.2019.00121

28. Mantel N, Haenszel W. Statistical Aspects of the Analysis of Data From Retrospective Studies of Disease. J Natl Cancer Inst (1959) 22(4):719-48.

29. DerSimonian R, Laird N. Meta-Analysis in Clinical Trials. Control Clin Trials (1986) 7(3):177-88. doi: 10.1016/0197-2456(86)90046-2

30. Salanti G. Indirect and Mixed-Treatment Comparison, Network, or MultipleTreatments Meta-Analysis: Many Names, Many Benefits, Many Concerns for the Next Generation Evidence Synthesis Tool. Res Synth Methods (2012) 3 (2):80-97. doi: 10.1002/jrsm.1037

31. Dias S, Welton NJ, Caldwell DM, Ades AE. Checking Consistency in Mixed Treatment Comparison Meta-Analysis. Stat Med (2010) 29(7-8):932-44. doi: 10.1002/sim.3767

32. Riley RD, Higgins JP, Deeks JJ. Interpretation of Random Effects MetaAnalyses. Bmj (2011) 342:d549. doi: 10.1136/bmj.d549

33. Guan Y, Liu ZB, Wang X, Liu YC, Chen GW. Analysis of Therapeutic Effects and Prognostic Factors for Hepatic Metastasis From Gastric Cancer. Med J Chin People's Liberation Army (2016) 41(4):312-6. doi: 10.11855/j.issn.05777402.2016.04.10

34. Kim HR, Ha Cheon S, Lee KH, Ryun Ahn J, Jeung HC, Sook Lee S, et al. Efficacy and Feasibility of Radiofrequency Ablation for Liver Metastases From Gastric Adenocarcinoma. Int J Hyperthermia (2010) 26(4):305-15. doi: 10.3109/02656730903555696

35. Li YM, Zhan WH, Han FH, He YL, Peng JS, Cai SR, et al. Clinicopathological Analysis of Synchronous Liver Metastasis in Gastric Cancer and Evaluation of Surgical Outcomes. Zhonghua Wei Chang Wai Ke Za Zhi = Chin J Gastrointest Surg (2006) 9(2):127-30.

36. Lu J, Wang WD, Xu P, Fan C. Investigation of the Therapeutic Regimes for Liver Metastasis From Gastric Cancer and Analysis of Therapeutic Effects. J Intervent Radiol (2010) 19(10):814-6.

37. Markar SR, Mackenzie H, Mikhail S, Mughal M, Preston SR, Maynard ND, et al. Surgical Resection of Hepatic Metastases From Gastric Cancer: Outcomes From National Series in England. Gastric Cancer (2017) 20 (2):379-86. doi: 10.1007/s10120-016-0604-6

38. Yao GL, Fan YG, Zhai JM, Lu BS, Liu KL. Radical Gastrectomy With Hepatoarterial Catheter Implantation for Late-Stage Gastric Cancer. World J Gastroenterol (2015) 21(9):2754-8. doi: 10.3748/wjg.v21.i9.2754

39. Shinohara T, Maeda Y, Hamada T, Futakawa N. Survival Benefit of Surgical Treatment for Liver Metastases From Gastric Cancer. J Gastrointest Surg (2015) 19(6):1043-51. doi: 10.1007/s11605-015-2775-6
40. Ohkura Y, Shinohara H, Haruta S, Ueno M, Hashimoto M, Sakai Y, et al. Hepatectomy Offers Superior Survival Compared With Non-Surgical Treatment for $</=3$ Metastatic Tumors With Diameters $<3 \mathrm{Cm}$ From Gastric Cancer: A Retrospective Study. World J Surg (2015) 39(11):2757-63. doi: $10.1007 / \mathrm{s} 00268-015-3151-0$

41. Liu SF, Lu CR, Cheng HD, Xi HQ, Cui JX, Li JY, et al. Comparison of Therapeutic Efficacy Between Gastrectomy With Transarterial Chemoembolization Plus Systemic Chemotherapy and Systemic Chemotherapy Alone in Gastric Cancer With Synchronous Liver Metastasis. Chin Med J (2015) 128(16):2194-201. doi: 10.4103/0366-6999.162497

42. Li Z, Fan B, Shan F, Tang L, Bu Z, Wu A, et al. Gastrectomy in Comprehensive Treatment of Advanced Gastric Cancer With Synchronous Liver Metastasis: A Prospectively Comparative Study. World J Surg Oncol (2015) 13:21261. doi: 10.1186/s12957-015-0627-1

43. Tiberio GAM, Baiocchi GL, Morgagni P, Marrelli D, Marchet A, Cipollari C, et al. Gastric Cancer and Synchronous Hepatic Metastases: Is It Possible to Recognize Candidates to R0 Resection? Ann Surg Oncol (2014) 22(2):589-96. doi: 10.1245/s10434-014-4018-6

44. Wang W, Liang H, Zhang H, Wang X, Xue Q, Zhang R. Prognostic Significance of Radical Surgical Treatment for Gastric Cancer Patients With Synchronous Liver Metastases. Med Oncol (2014) 31(11):1-8. doi: 10.1007/ s12032-014-0258-3

45. Chen L, Song MQ, Lin HZ, Hao LH, Jiang XJ, Li ZY, et al. Chemotherapy and Resection for Gastric Cancer With Synchronous Liver Metastases. World J Gastroenterol (2013) 19(13):2097-103. doi: 10.3748/wjg.v19.i13.2097

46. Miki Y, Fujitani K, Hirao M, Kurokawa Y, Mano M, Tsujie M, et al. Significance of Surgical Treatment of Liver Metastases From Gastric Cancer. Anticancer Res (2012) 32(2):665-70.

47. Makino H, Kunisaki C, Izumisawa $Y$, Tokuhisa M, Oshima T, Nagano Y, et al. Indication for Hepatic Resection in the Treatment of Liver Metastasis From Gastric Cancer. Anticancer Res (2010) 30(6):2367-76.

48. Cheon SH, Rha SY, Jeung HC, Im CK, Kim SH, Kim HR, et al. Survival Benefit of Combined Curative Resection of the Stomach (D2 Resection) and Liver in Gastric Cancer Patients With Liver Metastases. Ann Oncol (2008) 19(6):114653. doi: $10.1093 /$ annonc/mdn026

49. Li SC, Lee CH, Hung CL, Wu JC, Chen JH. Surgical Resection of Metachronous Hepatic Metastases From Gastric Cancer Improves LongTerm Survival: A Population-Based Study. PloS One (2017) 12(7):e0182255. doi: 10.1371/journal.pone.0182255

50. Ryu T, Takami Y, Wada Y, Tateishi M, Matsushima H, Yoshitomi M, et al. Oncological Outcomes After Hepatic Resection and/or Surgical Microwave Ablation for Liver Metastasis From Gastric Cancer. Asian J Surg (2019) 42 (1):100-5. doi: 10.1016/j.asjsur.2017.09.005

51. Shirasu H, Tsushima T, Kawahira M, Kawai S, Kawakami T, Kito Y, et al. Role of Hepatectomy in Gastric Cancer With Multiple Liver-Limited Metastases. Gastric Cancer (2018) 21(2):338-44. doi: 10.1007/s10120-017-0730-9

52. Tiberio GAM, Coniglio A, Marchet A, Marrelli D, Giacopuzzi S, Baiocchi L, et al. Metachronous Hepatic Metastases From Gastric Carcinoma: A Multicentric Survey. Eur J Surg Oncol (2009) 35(5):486-91. doi: 10.1016/j.ejso.2008.12.017

53. Zhou B, He N, Hong J, Yang T, Ng DM, Gao X, et al. HIFU for the Treatment of Gastric Cancer With Liver Metastases With Unsuitable Indications for Hepatectomy and Radiofrequency Ablation: A Prospective and Propensity Score-Matched Study. BMC Surg (2021) 21(1):308. doi: 10.1186/s12893-02101307-y

54. Jagric T, Horvat M. Surgical Resection of Synchronous Liver Metastases in Gastric Cancer Patients. A Propensity Score-Matched Study. Radiol Oncol (2020) 55(1):57-65. doi: 10.2478/raon-2020-0067

55. Tang K, Zhang B, Dong L, Wang L, Tang Z. Radiofrequency Ablation Versus Traditional Liver Resection and Chemotherapy for Liver Metastases From Gastric Cancer. J Int Med Res (2020) 48(7):300060520940509. doi: 10.1177/ 0300060520940509

56. Yu P, Zhang Y, Ye Z, Chen X, Huang L, Du Y, et al. Treatment of Synchronous Liver Metastases From Gastric Cancer: A Single-Center Study. Cancer Manage Res (2020) 12:7905-11. doi: 10.2147/cmar.S261353

57. Picado O, Dygert L, Macedo FI, Franceschi D, Sleeman D, Livingstone AS, et al. The Role of Surgical Resection for Stage IV Gastric Cancer With Synchronous Hepatic Metastasis. J Surg Res (2018) 232:422-9. doi: 10.1016/ j.jss.2018.06.067 
58. Wang W, Liang H, Zhang H, Wang X, Xue Q, Zhang R. Prognostic Significance of Radical Surgical Treatment for Gastric Cancer Patients With Synchronous Liver Metastases. Med Oncol (Northwood London England) (2014) 31(11):258. doi: 10.1007/s12032-014-0258-3

59. Kim KH, Lee K, Baek SK, Chang HJ, Kim YJ, Park DJ, et al. Survival Benefit of Gastrectomy \pm Metastasectomy in Metastatic Gastric Cancer Patients Receiving Chemotherapy. Ann Oncol (2010) 21:viii231. doi: 10.1093/ annonc/mdq522

60. Tsujimoto H, Ichikura T, Ono S, Sugasawa H, Hiraki S, Sakamoto N, et al. Outcomes for Patients Following Hepatic Resection of Metastatic Tumors From Gastric Cancer. Hepatol Int (2010) 4(1):406-13. doi: 10.1007/s12072009-9161-y

61. Song A, Zhang X, Yu F, Li D, Shao W, Zhou Y. Surgical Resection for Hepatic Metastasis From Gastric Cancer: A Multi-Institution Study. Oncotarget (2017) 8:71147-53. doi: 10.18632/oncotarget.16705

62. Murthy R, Xiong H, Nunez R, Cohen AC, Barron B, Szklaruk J, et al. Yttrium 90 Resin Microspheres for the Treatment of Unresectable Colorectal Hepatic Metastases After Failure of Multiple Chemotherapy Regimens: Preliminary Results. J Vasc Intervent Radiol: JVIR (2005) 16(7):937-45. doi: 10.1097/ 01.rvi.0000161142.12822.66

63. Cercek A, Boucher TM, Gluskin JS, Aguilo A, Chou JF, Connell LC, et al. Response Rates of Hepatic Arterial Infusion Pump Therapy in Patients With Metastatic Colorectal Cancer Liver Metastases Refractory to All Standard Chemotherapies. J Surg Oncol (2016) 114(6):655-63. doi: 10.1002/jso.24399

64. Zampino MG, Magni E, Ravenda PS, Cella CA, Bonomo G, Della Vigna P, et al. Treatments for Colorectal Liver Metastases: A New Focus on a Familiar Concept. Crit Rev Oncol Hematol (2016) 108:154-63. doi: 10.1016/ j.critrevonc.2016.11.005

65. Nosher JL, Ahmed I, Patel AN, Gendel V, Murillo PG, Moss R, et al. NonOperative Therapies for Colorectal Liver Metastases. J Gastrointest Oncol (2015) 6(2):224-40. doi: 10.3978/j.issn.2078-6891.2014.065

66. Assumpcao L, Choti MA, Gleisner AL, Schulick RD, Swartz M, Herman J, et al. Patterns of Recurrence Following Liver Resection for Colorectal Metastases: Effect of Primary Rectal Tumor Site. Arch Surg (Chicago Ill: 1960) (2008) 143(8):743-9; discussion 9-50. doi: 10.1001/archsurg.143.8.743

67. de Jong GM, Hendriks T, Eek A, Oyen WJ, Nagtegaal ID, Bleichrodt RP, et al. Adjuvant Radioimmunotherapy Improves Survival of Rats After Resection of Colorectal Liver Metastases. Ann Surg (2011) 253(2):336-41. doi: 10.1097/ SLA.0b013e3181ff313a

68. Fiorentini G, Poddie DB, Cantore M, Giovanis P, Guadagni S, De Giorgi U, et al. Locoregional Therapy for Liver Metastases From Colorectal Cancer: The Possibilities of Intraarterial Chemotherapy, and New Hepatic-Directed Modalities. Hepatogastroenterology (2001) 48(38):305-12.

69. Fiorentini G, Poddie DB, De Giorgi U, Guglielminetti D, Giovanis P, Leoni M, et al. Global Approach to Hepatic Metastases From Colorectal Cancer: Indication and Outcome of Intra-Arterial Chemotherapy and Other Hepatic-Directed Treatments. Med Oncol (Northwood London England) (2000) 17(3):163-73. doi: 10.1007/BF02780523

70. Gleisner AL, Choti MA, Assumpcao L, Nathan H, Schulick RD, Pawlik TM. Colorectal Liver Metastases: Recurrence and Survival Following Hepatic Resection, Radiofrequency Ablation, and Combined ResectionRadiofrequency Ablation. Arch Surg (Chicago Ill: 1960) (2008) 143 (12):1204-12. doi: 10.1001/archsurg.143.12.1204

71. Gulec SA. Y-90 Radiomicrosphere Therapy for Colorectal Cancer Liver Metastases. Semin Nucl Med (2016) 46(2):126-34. doi: 10.1053/ j.semnuclmed.2015.10.008

72. Hughes KS, Simon R, Songhorabodi S, Adson MA, Ilstrup DM, Fortner JG, et al. Resection of the Liver for Colorectal Carcinoma Metastases: A MultiInstitutional Study of Patterns of Recurrence. Surgery (1986) 100(2):278-84.

73. Maleux G, Deroose C, Laenen A, Verslype C, Heye S, Haustermans K, et al. Yttrium-90 Radioembolization for the Treatment of Chemorefractory Colorectal Liver Metastases: Technical Results, Clinical Outcome and Factors Potentially Influencing Survival. Acta Oncol (Stockholm Sweden) (2016) 55(4):486-95. doi: 10.3109/0284186X.2015.1101151

74. Sag AA, Selcukbiricik F, Mandel NM. Evidence-Based Medical Oncology and Interventional Radiology Paradigms for Liver-Dominant Colorectal Cancer
Metastases. World J Gastroenterol (2016) 22(11):3127-49. doi: 10.3748/ wjg.v22.i11.3127

75. Scheele J, Altendorf-Hofmann A. Resection of Colorectal Liver Metastases. Langenbecks Arch Surg (1999) 384(4):313-27. doi: 10.1007/s004230050209

76. Tanaka K, Murakami T, Yabushita Y, Hiroshima Y, Matsuo K, Endo I, et al. Maximal Debulking Liver Resection as a Beneficial Treatment Strategy for Advanced and Aggressive Colorectal Liver Metastases. Anticancer Res (2014) 34(10):5547-54.

77. Vogl TJ, Emam A, Naguib NN, Eichler K, Zangos S. How Effective Are Percutaneous Liver-Directed Therapies in Patients With Non-Colorectal Liver Metastases? Viszeralmedizin (2015) 31(6):406-13. doi: 10.1159/000440677

78. Wiggans MG, Shahtahmassebi G, Aroori S, Bowles MJ, Briggs C, Stell DA. The Pre-Operative Rate of Growth of Colorectal Metastases in Patients Selected for Liver Resection Does Not Influence Post-Operative DiseaseFree Survival. Eur J Surg Oncol: J Eur Soc Surg Oncol Br Assoc Surg Oncol (2016) 42(3):426-32. doi: 10.1016/j.ejso.2015.09.016

79. Luo Z, Rong Z, Huang C. Surgery Strategies for Gastric Cancer With Liver Metastasis. Front Oncol (2019) 9:1353. doi: 10.3389/fonc.2019.01353

80. Leporrier J, Maurel J, Chiche L, Bara S, Segol P, Launoy G. A PopulationBased Study of the Incidence, Management and Prognosis of Hepatic Metastases From Colorectal Cancer. Br J Surg (2006) 93(4):465-74. doi: $10.1002 /$ bjs. 5278

81. Kinoshita T, Kinoshita T, Saiura A, Esaki M, Sakamoto H, Yamanaka T. Multicentre Analysis of Long-Term Outcome After Surgical Resection for Gastric Cancer Liver Metastases. Br J Surg (2015) 102(1):102-7. doi: 10.1002/ bjs. 9684

82. Tiberio GAM, Ferrari VD, Ministrini S, Cipollari C, Graziosi L, Morgagni P, et al. Gastric Cancer With Synchronous Hepatic Metastases: A Multicentric Italian Survey. J Clin Oncol (2013) 31(15). doi: 10.1200/jco.2013.31.15_suppl.e15148

83. Qiu JL, Deng MG, Li W, Zou RH, Li BK, Zheng Y, et al. Hepatic Resection for Synchronous Hepatic Metastasis From Gastric Cancer. Eur J Surg Oncol (2013) 39(7):694-700. doi: 10.1016/j.ejso.2013.03.006

84. Liu P, Zhu X, Yang RJ, Chen H, Xu HF, Gao S, et al. Interventional Treatment for Progressive Hepatic Metastases From Gastric Cancer After Chemotherapy: A Retrospective Analysis of 72 Cases. J Intervent Radiol (China) (2013) 22 (9):742-6. doi: 10.3969/j.issn.1008-794X.2013.09.010

85. Baek HU, Kim SB, Cho EH, Jin SH, Yu HJ, Lee JI, et al. Hepatic Resection for Hepatic Metastases From Gastric Adenocarcinoma. J Gastric Cancer (2013) 13 (2):86-92. doi: 10.5230/jgc.2013.13.2.86

86. Zhang K, Chen L. Chinese Consensus on the Diagnosis and Treatment of Gastric Cancer With Liver Metastases. Ther Adv Med Oncol (2020) 12:1758835920904803. doi: 10.1177/1758835920904803

87. Liao YY, Peng NF, Long D, Yu PC, Zhang S, Zhong JH, et al. Hepatectomy for Liver Metastases From Gastric Cancer: A Systematic Review. BMC Surg (2017) 17(1):14. doi: 10.1186/s12893-017-0215-0

88. Wang X, Sofocleous CT, Erinjeri JP, Petre EN, Gonen M, Do KG, et al. Margin Size is an Independent Predictor of Local Tumor Progression After Ablation of Colon Cancer Liver Metastases. Cardiovasc Intervent Radiol (2013) 36 (1):166-75. doi: 10.1007/s00270-012-0377-1

Conflict of Interest: The authors declare that the research was conducted in the absence of any commercial or financial relationships that could be construed as a potential conflict of interest.

Publisher's Note: All claims expressed in this article are solely those of the authors and do not necessarily represent those of their affiliated organizations, or those of the publisher, the editors and the reviewers. Any product that may be evaluated in this article, or claim that may be made by its manufacturer, is not guaranteed or endorsed by the publisher.

Copyright (c) 2021 Sun, Ding, Zhu, Wang, Gu, Xia and Li. This is an open-access article distributed under the terms of the Creative Commons Attribution License (CC BY). The use, distribution or reproduction in other forums is permitted, provided the original author(s) and the copyright owner(s) are credited and that the original publication in this journal is cited, in accordance with accepted academic practice. No use, distribution or reproduction is permitted which does not comply with these terms. 